\title{
One-loop corrections to light neutrino masses in gauged U(1) extensions of the standard model
}

\author{
Sho Iwamoto๑* and Timo J. Kärkkäinen $\oplus^{\dagger}$ \\ Institute for Theoretical Physics, ELTE Eötvös Loránd University, \\ Pázmány Péter sétány 1/A, 1117 Budapest, Hungary \\ Zoltán Péli $\oplus^{\ddagger}$ \\ ELKH-DE Particle Physics Research Group, 4010 Debrecen, P.O. Box 105, Hungary \\ Zoltán Trócsányi $\oplus^{\S}$ \\ Institute for Theoretical Physics, ELTE Eötvös Loránd University, Pázmány Péter sétány 1/A, \\ 1117 Budapest, Hungary and ELKH-DE Particle Physics Research Group, \\ 4010 Debrecen, P.O. Box 105, Hungary
}

(Received 10 May 2021; accepted 19 August 2021; published 28 September 2021)

\begin{abstract}
We consider gauged U(1) extensions of the standard model of particle physics with three right-handed sterile neutrinos and a singlet scalar. The neutrinos obtain mass via the type I seesaw mechanism. We compute the one-loop corrections to the elements of the tree-level mass matrix of the light neutrinos and show explicitly the cancellation of the gauge-dependent terms. We present a general formula for the gaugeindependent, finite one-loop corrections for arbitrary number new U(1) groups, new complex scalars, and sterile neutrinos. We estimate the size of the corrections relative to the tree-level mass matrix in a particular extension, the superweak model.
\end{abstract}

DOI: $10.1103 /$ PhysRevD.104.055042

\section{INTRODUCTION}

The standard model (SM) of particle interactions is one of the most successful physics models with unprecedented precision for predicting physical quantities, for instance, for the anomalous magnetic moment of the electron. However, it does not contain right-handed neutrinos as they are sterile under the SM gauge group. This in turn leads to the prediction that neutrinos are massless, which is in conflict with the now well-established experimental result, that at least two neutrinos are massive [1,2], and therefore, signals that the SM requires an extension to explain the origin of the neutrino masses. There are lots of models attempting to explain neutrino masses. Among those, perhaps the most economical one that requires the least extension of the SM is the type I seesaw mechanism where neutrinos acquire

\footnotetext{
*sho.iwamoto@ttk.elte.hu

timo.karkkainen@ttk.elte.hu

zoltanpeli92@gmail.com

${ }^{\S}$ zoltan.trocsanyi@cern.ch
}

Published by the American Physical Society under the terms of the Creative Commons Attribution 4.0 International license. Further distribution of this work must maintain attribution to the author(s) and the published article's title, journal citation, and DOI. Funded by SCOAP ${ }^{3}$. masses after spontaneous symmetry breaking (SSB) of one or more scalar fields [3-10].

Recently, there has been a lot of interest in gauged $\mathrm{U}(1)$-extended models in particle physics phenomenology motivated by the observed difference between the measured and SM predicted values of the anomalous magnetic moment of the muon [11] and also anomalies in shortbaseline neutrino oscillations [12]. Gauged $B-L$, $B-3 L_{\tau}, L_{e}-L_{\mu}$, and $L_{\mu}-L_{\tau}$ have been considered [13-19], as well as a general gauged U(1) not related to flavor [20]. In these models, both seesaw and radiative oneloop neutrino mass generation mechanisms have been considered.

As the effects of new physics are typically much smaller than those of the SM interactions, computations in theories beyond the SM are often considered only at tree level. Yet, the loop corrections may be sizable and can affect significantly the validity region in the parameter space of the model. For instance, the lightness of active neutrinos requires that the loop corrections to the mass matrix of those particles must also be small in order to have a phenomenologically viable model. Computations of radiatively induced neutrino masses were performed first within the standard model in Ref. [21], while one-loop corrections to tree-level masses have been obtained previously in Refs. [22-24] for the canonical seesaw case, in 
Ref. [25] for the inverse seesaw case, and in the context of multi-Higgs doublet models [26-29]. In the cases of gauged $\mathrm{U}(1)$ models, we are not aware of a computation of the one-loop corrections to the active neutrino mass matrix.

In this article, we consider gauged $\mathrm{U}(1)$ extensions of the SM and derive a general formula for the one-loop corrections of the mass matrix of the active neutrinos. The mass matrix of the active neutrinos emerges after SSB due to the type I seesaw mechanism. Our goal is to derive the oneloop corrections to that mass matrix and estimate their sizes relative to the tree level for a particular example called the superweak force [30]. The superweak model contains three additional right-handed sterile (under the SM interactions) neutrinos and one complex scalar field in addition to the fields of the SM. The loop corrections involve all the gauge and scalar bosons which couple to neutrinos.

In order to obtain the one-loop corrections to the elements of the light neutrino mass matrix, we perform our computations in the $R_{\xi}$ gauge and show explicitly the intricate cancellation of the gauge fixing parameters from the corrections. In addition, we also demonstrate the cancellation of the $\epsilon$ poles when the loop integrals are regulated by dimensional regularization in $d=4-2 \epsilon$ dimensions. These cancellations are highly nontrivial, and therefore provide strong checks on the correctness of the computations.

The paper is composed as follows. We introduce the model to the extent needed for the present work in Sec. II. We define and compute the one-loop correction to the mass matrix of the active neutrinos in Sec. III. In Sec. IV, we provide numerical estimates of the one-loop corrections and show that those are very small. Finally, we summarize our findings in Sec. V. We collect auxiliary formulas in the Appendixes and also provide an auxiliary zip file containing the SARAH model, parameter, and particle files.

\section{PARTICLE MODEL, MIXINGS, AND INTERACTIONS}

We consider an extension of the standard model by a $\mathrm{U}(1)_{z}$ gauge group with particle content and charge assignment defined in Ref. [30]. The superweak model is an economical extension of the standard model that provides a framework to explain the origin of (i) neutrino masses and oscillations [31], (ii) dark matter [32], (iii) cosmic inflation and stabilization of the electroweak vacuum [33], and (iv) matter-antimatter asymmetry of the Universe. The complete model including Feynman rules in the unitary gauge was presented fully in Ref. [30]. As we are to compute one-loop corrections to neutrino masses, we recall the details relevant to such computations, with Feynman rules in the $R_{\xi}$ gauge. We generated those Feynman rules with SARAH [34-36], but here we present simpler forms for the rules needed in our computations to make those more comprehensive. We also recall some of the conventions that are different in SARAH and the original definition of the model. We stick to the SARAH conventions throughout this work [37].

\section{A. Mixing of neutral gauge bosons}

The particle content of the standard model is extended by three right-handed neutrinos $\nu_{\mathrm{Ri}}$, a new scalar $\chi$, and the $\mathrm{U}(1)_{z}$ gauge boson $B^{\prime}$. As the field strength tensors of the $\mathrm{U}(1)$ gauge groups are gauge invariant, kinetic mixing is allowed between the gauge fields belonging to the hypercharge $\mathrm{U}(1)_{y}$ and the new $\mathrm{U}(1)_{z}$ gauge symmetries, whose strength is measured by $\epsilon$ in

$$
\begin{aligned}
& \mathcal{L} \supset-\frac{1}{4} F^{\mu \nu} F_{\mu \nu}-\frac{1}{4} F^{\prime \mu \nu} F_{\mu \nu}^{\prime}-\frac{\epsilon}{2} F^{\mu \nu} F_{\mu \nu}^{\prime}, \\
& \mathcal{D}_{\mu}^{\mathrm{U}(1)}=-\mathrm{i}\left(y g_{y} B_{\mu}+z g_{z} B_{\mu}^{\prime}\right),
\end{aligned}
$$

where $B^{\mu}$ is the $\mathrm{U}(1)_{y}$ gauge field. The charge assignments are shown in Table I (cf. Ref. [30]). The new fields are neutral under the standard model gauge interactions. The $y$ charges are the eigenvalues of half of the hypercharge operator. The $z$ charges are assigned such that gauge and gravity anomalies cancel in each family.

Equivalent to the kinetic mixing, we can choose the basis - the convention in SARAH - in which the gauge-field strengths do not mix, while the couplings are given by a $2 \times 2$ coupling matrix in the covariant derivative

$$
D_{\mu}^{\mathrm{U}(1)}=-\mathrm{i}\left(\begin{array}{ll}
y & z
\end{array}\right)\left(\begin{array}{cc}
\hat{g}_{y y} & \hat{g}_{y z} \\
\hat{g}_{z y} & \hat{g}_{z z}
\end{array}\right)\left(\begin{array}{c}
\hat{B}_{\mu} \\
\hat{B}_{\mu}^{\prime}
\end{array}\right)
$$

where $y$ and $z$ are the $\mathrm{U}(1)_{y}$ and $\mathrm{U}(1)_{z}$ charges. We can parametrize the coupling matrix as

TABLE I. Particle content and charge assignment of the superweak model, where $\phi$ and $\chi$ are complex scalars and the others are Weyl fermions. For $\mathrm{SU}(3)_{\mathrm{c}} \otimes \mathrm{SU}(2)_{\mathrm{L}}$, the representations are given, while for $\mathrm{U}(1)_{y} \otimes U(1)_{z}$, the charges $(y$ and $z$ ) of the respective fields are given. Note that for $\mathrm{U}(1)_{y}$, the eigenvalues of the half-hypercharge operator are given.

\begin{tabular}{lcccc}
\hline \hline & $\mathrm{SU}(3)_{\mathrm{c}}$ & $\mathrm{SU}(2)_{\mathrm{L}}$ & $\mathrm{U}(1)_{y}$ & $\mathrm{U}(1)_{z}$ \\
\hline$Q_{\mathrm{L}}$ & $\mathbf{3}$ & $\mathbf{2}$ & $1 / 6$ & $1 / 6$ \\
$U_{\mathrm{R}}$ & $\mathbf{3}$ & $\mathbf{1}$ & $2 / 3$ & $7 / 6$ \\
$D_{\mathrm{R}}$ & $\mathbf{3}$ & $\mathbf{1}$ & $-1 / 3$ & $-5 / 6$ \\
$L_{\mathrm{L}}$ & $\mathbf{1}$ & $\mathbf{2}$ & $-1 / 2$ & $-1 / 2$ \\
$N_{\mathrm{R}}$ & $\mathbf{1}$ & $\mathbf{1}$ & 0 & $1 / 2$ \\
$e_{\mathrm{R}}$ & $\mathbf{1}$ & $\mathbf{1}$ & -1 & $-3 / 2$ \\
$\phi$ & $\mathbf{1}$ & $\mathbf{2}$ & $1 / 2$ & 1 \\
$\chi$ & $\mathbf{1}$ & $\mathbf{1}$ & 0 & -1 \\
\hline \hline
\end{tabular}




$$
\hat{\mathbf{g}}=\left(\begin{array}{ll}
\hat{g}_{y y} & \hat{g}_{y z} \\
\hat{g}_{z y} & \hat{g}_{z z}
\end{array}\right)=\left(\begin{array}{cc}
g_{y} & -\eta g_{z}^{\prime} \\
0 & g_{z}^{\prime}
\end{array}\right)\left(\begin{array}{cc}
\cos \epsilon^{\prime} & \sin \epsilon^{\prime} \\
-\sin \epsilon^{\prime} & \cos \epsilon^{\prime}
\end{array}\right) .
$$

The coupling mixing matrix containing $\eta$ is equivalent to the kinetic mixing in the Lagrangian (2.1), and the parameters of the two representations are related by $g_{z}^{\prime}=g_{z} / \sqrt{1-\epsilon^{2}}$ and $\eta=\epsilon g_{y} / g_{z}$. In this paper, it will be convenient to use the kinetic mixing representation defined by $(2.1)$.

The rotation with angle $\epsilon^{\prime}$ is unphysical, as it can be absorbed into the mixing of the neutral gauge fields $B^{\mu}, B^{\prime \mu}$, and $W^{3 \mu}$ to the mass eigenstates $A^{\mu}, Z^{\mu}$, and $Z^{\prime \mu}$, which then can be described by a rotation matrix

$$
\begin{aligned}
\left(\begin{array}{c}
\hat{B}^{\mu} \\
W^{3 \mu} \\
\hat{B}^{\prime \mu}
\end{array}\right)= & \left(\begin{array}{ccc}
\cos \theta_{\mathrm{W}} & -\cos \theta_{Z} \sin \theta_{\mathrm{W}} & -\sin \theta_{Z} \sin \theta_{\mathrm{W}} \\
\sin \theta_{\mathrm{W}} & \cos \theta_{Z} \cos \theta_{\mathrm{W}} & \cos \theta_{\mathrm{W}} \sin \theta_{Z} \\
0 & -\sin \theta_{Z} & \cos \theta_{Z}
\end{array}\right) \\
& \times\left(\begin{array}{c}
A^{\mu} \\
Z^{\mu} \\
Z^{\prime \mu}
\end{array}\right)
\end{aligned}
$$

This matrix depends on two mixing angles: $\theta_{\mathrm{W}}$ is the weak mixing (or Weinberg) angle and $\theta_{Z}$ is the $Z-Z^{\prime}$ mixing angle [38]. In terms of the coupling parameters

$\kappa=\cos \theta_{\mathrm{W}}\left(\gamma_{y}^{\prime}-2 \gamma_{z}^{\prime}\right) \quad$ and $\quad \tau=2 \cos \theta_{\mathrm{W}} \gamma_{z}^{\prime} \tan \beta$

introduced in Ref. [30], this new mixing angle is given implicitly by $\tan \left(2 \theta_{Z}\right)=2 \kappa /\left(1-\kappa^{2}-\tau^{2}\right)$. In Eq. (2.5), $\tan \beta=w / v$ is the ratio of the vacuum expectation values (VEVs) of the scalar fields (see below) and $\gamma_{y}^{\prime}=\left(\epsilon / \sqrt{1-\epsilon^{2}}\right)\left(g_{y} / g_{\mathrm{L}}\right), \gamma_{z}^{\prime}=g_{z}^{\prime} / g_{\mathrm{L}}$; i.e., the couplings are normalized by the $\mathrm{SU}(2)_{\mathrm{L}}$ coupling.

We can express the elements of the $Z-Z^{\prime}$ mixing matrix explicitly,

$$
\begin{aligned}
& \sin \theta_{Z}=\operatorname{sgn}(\kappa)\left[\frac{1}{2}\left(1-\frac{1-\kappa^{2}-\tau^{2}}{\sqrt{\left(1+\kappa^{2}+\tau^{2}\right)^{2}-4 \tau^{2}}}\right)\right]^{1 / 2} \\
& \cos \theta_{Z}=\left[\frac{1}{2}\left(1+\frac{1-\kappa^{2}-\tau^{2}}{\sqrt{\left(1+\kappa^{2}+\tau^{2}\right)^{2}-4 \tau^{2}}}\right)\right]^{1 / 2}
\end{aligned}
$$

which also appear in the neutral currents $\Gamma_{V \bar{f} f}^{\mu}=$ $-\mathrm{i} e \gamma^{\mu}\left(C_{V \bar{f} f}^{R} P_{R}+C_{V \bar{f} f}^{L} P_{L}\right)$ where $e$ is the electromagnetic coupling and $P_{R / L} \equiv P_{ \pm}=\frac{1}{2}\left(1 \pm \gamma^{5}\right)$ are the usual chiral projections. In particular, for neutrinos $e C_{Z \nu \nu}^{L}=\frac{g_{\mathrm{L}}}{2 \cos \theta_{\mathrm{W}}}\left[\cos \theta_{Z}-\left(\gamma_{y}^{\prime}-\gamma_{z}^{\prime}\right) \sin \theta_{Z} \cos \theta_{\mathrm{W}}\right]$,

$e C_{Z \nu \nu}^{R}=-\frac{g_{\mathrm{L}}}{2} \gamma_{z}^{\prime} \sin \theta_{Z}$,

$e C_{Z^{\prime} \nu \nu}^{L}=\frac{g_{\mathrm{L}}}{2 \cos \theta_{\mathrm{W}}}\left[\sin \theta_{Z}+\left(\gamma_{y}^{\prime}-\gamma_{z}^{\prime}\right) \cos \theta_{Z} \cos \theta_{\mathrm{W}}\right]$,

$e C_{Z^{\prime} \nu \nu}^{R}=\frac{g_{\mathrm{L}}}{2} \gamma_{z}^{\prime} \cos \theta_{Z}$;

i.e., $C_{Z^{\prime} \nu \nu}^{L / R}$ can be obtained from $C_{Z \nu \nu}^{L / R}$ by the replacement

$\left(Z \rightarrow Z^{\prime}\right) \Rightarrow\left(\cos \theta_{Z}, \sin \theta_{Z}\right) \rightarrow\left(\sin \theta_{Z},-\cos \theta_{Z}\right)$.

\section{B. Mixings of scalar and Goldstone bosons}

In addition to the usual $\mathrm{SU}(2)_{\mathrm{L}}$-doublet Brout-EnglertHiggs field

$$
\phi=\left(\begin{array}{c}
\phi^{+} \\
\phi^{0}
\end{array}\right)=\frac{1}{\sqrt{2}}\left(\begin{array}{c}
\phi_{1}+\mathrm{i} \phi_{2} \\
\phi_{3}+\mathrm{i} \phi_{4}
\end{array}\right)
$$

there is another complex scalar $\chi$ in the model with charges specified in [30]. The Lagrangian of the scalar fields contains the potential energy

$$
\begin{aligned}
V(\phi, \chi)= & V_{0}-\mu_{\phi}^{2}|\phi|^{2}-\mu_{\chi}^{2}|\chi|^{2} \\
& +\left(|\phi|^{2},|\chi|^{2}\right)\left(\begin{array}{cc}
\lambda_{\phi} & \frac{\lambda}{2} \\
\frac{\lambda}{2} & \lambda_{\chi}
\end{array}\right)\left(\begin{array}{c}
|\phi|^{2} \\
|\chi|^{2}
\end{array}\right) \subset-\mathcal{L},
\end{aligned}
$$

where $|\phi|^{2}=\left|\phi^{+}\right|^{2}+\left|\phi^{0}\right|^{2}$. In the $R_{\xi}$ gauge, we parametrize the scalar fields after spontaneous symmetry breaking as

$$
\begin{aligned}
& \phi=\frac{1}{\sqrt{2}}\left(\begin{array}{c}
-\mathrm{i} \sqrt{2} \sigma^{+} \\
v+h^{\prime}+\mathrm{i} \sigma_{\phi}
\end{array}\right), \\
& \chi=\frac{1}{\sqrt{2}}\left(w+s^{\prime}+\mathrm{i} \sigma_{\chi}\right),
\end{aligned}
$$

where $v$ and $w$ denote the VEVs of the fields, whose values are

$$
\begin{aligned}
& v=\sqrt{2} \sqrt{\frac{2 \lambda_{\chi} \mu_{\phi}^{2}-\lambda \mu_{\chi}^{2}}{4 \lambda_{\phi} \lambda_{\chi}-\lambda^{2}}}, \\
& w=\sqrt{2} \sqrt{\frac{2 \lambda_{\phi} \mu_{\chi}^{2}-\lambda \mu_{\phi}^{2}}{4 \lambda_{\phi} \lambda_{\chi}-\lambda^{2}}} .
\end{aligned}
$$

Using the VEVs, we can express the quadratic couplings as

$$
\mu_{\phi}^{2}=\lambda_{\phi} v^{2}+\frac{\lambda}{2} w^{2}, \quad \mu_{\chi}^{2}=\lambda_{\chi} w^{2}+\frac{\lambda}{2} v^{2} .
$$

The fields $h^{\prime}$ and $s^{\prime}$ are two real scalars, and $\sigma_{\phi}$ and $\sigma_{\chi}$ are the corresponding Goldstone bosons that are weak 
eigenstates. We shall denote the mass eigenstates with $h, s$ and $\sigma_{Z}, \sigma_{Z^{\prime}}$. These different eigenstates are related by the rotations

$$
\left(\begin{array}{l}
h \\
s
\end{array}\right)=\mathbf{Z}_{S}\left(\begin{array}{c}
h^{\prime} \\
s^{\prime}
\end{array}\right) \equiv\left(\begin{array}{cc}
\cos \theta_{S} & -\sin \theta_{S} \\
\sin \theta_{S} & \cos \theta_{S}
\end{array}\right)\left(\begin{array}{c}
h^{\prime} \\
s^{\prime}
\end{array}\right)
$$

and

$$
\left(\begin{array}{c}
\sigma_{Z} \\
\sigma_{Z^{\prime}}
\end{array}\right)=\mathbf{Z}_{\mathrm{G}}\left(\begin{array}{c}
\sigma_{\phi} \\
\sigma_{\chi}
\end{array}\right) \equiv\left(\begin{array}{cc}
\cos \theta_{\mathrm{G}} & -\sin \theta_{\mathrm{G}} \\
\sin \theta_{\mathrm{G}} & \cos \theta_{\mathrm{G}}
\end{array}\right)\left(\begin{array}{c}
\sigma_{\phi} \\
\sigma_{\chi}
\end{array}\right)
$$

where $\theta_{S}$ and $\theta_{\mathrm{G}}$ are the scalar and Goldstone mixing angles that can be determined by the diagonalization of the mass matrix of the real scalars and that of the neutral Goldstone bosons.

The scalar mixing angle $\theta_{S}$ is related to the potential parameters by [30]

$$
\tan \left(2 \theta_{S}\right)=-\frac{\lambda v w}{\lambda_{\phi} v^{2}-\lambda_{\chi} w^{2}}
$$

The condition $\theta_{S} \in\left(-\frac{\pi}{4}, \frac{\pi}{4}\right)$ implies that the scalar mass eigenstates are not labeled by mass hierarchy.

The mass matrix of the Goldstone bosons is given in principle by the sum of gauge-independent and gaugedependent terms. However, the gauge-independent terms vanish by Eq. (2.13):

$$
\left(\begin{array}{cc}
\frac{1}{2} \lambda w^{2}+\lambda_{\phi} v^{2}-\mu_{\phi}^{2} & 0 \\
0 & \frac{1}{2} \lambda v^{2}+\lambda_{\chi} w^{2}-\mu_{\chi}^{2}
\end{array}\right)=\mathbf{0},
$$

so the mass matrix contains only gauge-dependent terms,

$$
\mathbf{m}_{A}^{2}=\xi_{Z} \mathbf{m}_{A_{Z}}^{2}+\xi_{Z^{\prime}} \mathbf{m}_{A_{Z^{\prime}}}^{2}
$$

where $\xi_{Z}$ and $\xi_{Z^{\prime}}$ are the gauge parameters. The mass matrix is symmetric, so we can write it formally as

$$
\mathbf{m}_{A_{x}}^{2}=\left(\begin{array}{ll}
m_{A_{x}, 11}^{2} & m_{A_{x}, 12}^{2} \\
m_{A_{x}, 12}^{2} & m_{A_{x}, 22}^{2}
\end{array}\right)
$$

for both $x=Z$ and $Z^{\prime}$. Explicitly,

$$
\begin{aligned}
m_{A_{Z}, 11}^{2} & =v^{2} e^{2}\left(C_{Z \nu \nu}^{L}-C_{Z \nu \nu}^{R}\right)^{2} \\
& =\left(\frac{M_{W}}{\cos \theta_{\mathrm{W}}}\right)^{2}\left(\cos \theta_{Z}-\kappa \sin \theta_{Z}\right)^{2}, \\
m_{A_{Z}, 12}^{2} & =2 v w e^{2}\left(C_{Z \nu \nu}^{L}-C_{Z \nu \nu}^{R}\right) C_{Z \nu \nu}^{R} \\
& =\left(\frac{M_{W}}{\cos \theta_{\mathrm{W}}}\right)^{2}\left(\cos \theta_{Z}-\kappa \sin \theta_{Z}\right)\left(-\tau \sin \theta_{Z}\right), \\
m_{A_{Z}, 22}^{2} & =w^{2} e^{2}\left(2 C_{Z \nu \nu}^{R}\right)^{2}=\left(\frac{M_{W}}{\cos \theta_{\mathrm{W}}}\right)^{2}\left(-\tau \sin \theta_{Z}\right)^{2},
\end{aligned}
$$

where $M_{W}=\frac{v g_{\mathrm{L}}}{2}$ is the mass of the $\mathrm{W}$ bosons, and the elements of $\mathbf{m}_{A_{Z^{\prime}}}^{2}$ can be obtained by the replacement $Z \rightarrow Z^{\prime}$ in the chiral couplings, which implies the replacement (2.8) in the second forms of the matrix elements. The latter are the most convenient ones for the diagonalization of the mass matrix. Using Eq. (2.6), one can check that the matrix

$$
\mathbf{Z}_{\mathrm{G}} \mathbf{m}_{A}^{2} \mathbf{Z}_{\mathrm{G}}^{T}=\mathbf{m}_{\text {diag }, A}^{2}
$$

is indeed diagonal provided we have for the Goldstone mixing angle

$$
\cos \theta_{\mathrm{G}}=\frac{\cos \theta_{Z}-\kappa \sin \theta_{Z}}{\sqrt{\left(\cos \theta_{Z}-\kappa \sin \theta_{Z}\right)^{2}+\left(\tau \sin \theta_{Z}\right)^{2}}}
$$

and

$$
\sin \theta_{\mathrm{G}}=\frac{\tau \sin \theta_{Z}}{\sqrt{\left(\cos \theta_{Z}-\kappa \sin \theta_{Z}\right)^{2}+\left(\tau \sin \theta_{Z}\right)^{2}}} .
$$

\section{Masses of neutral gauge bosons}

As expected, the elements of the diagonal matrix $\mathbf{m}_{\text {diag, }, A}^{2}$ coincide with the squares of the masses of the neutral gauge bosons [30],

$M_{Z}^{2}=\left(\frac{M_{W}}{\cos \theta_{\mathrm{W}}}\right)^{2}\left[\left(\cos \theta_{Z}-\kappa \sin \theta_{Z}\right)^{2}+\left(\tau \sin \theta_{Z}\right)^{2}\right]$

and

$M_{Z^{\prime}}^{2}=\left(\frac{M_{W}}{\cos \theta_{\mathrm{W}}}\right)^{2}\left[\left(\sin \theta_{Z}+\kappa \cos \theta_{Z}\right)^{2}+\left(\tau \cos \theta_{Z}\right)^{2}\right]$,

which can also be expressed conveniently with the chiral couplings and Goldstone mixing angle. First we note that using Eq. (2.23), we find the simple relation

$$
\sin \theta_{\mathrm{G}}=\tau \frac{\sin \theta_{\mathrm{Z}}}{\cos \theta_{\mathrm{W}}} \frac{M_{W}}{M_{Z}}
$$

between the Goldstone and neutral boson mixing angles, and also 


$$
\cos \theta_{\mathrm{G}}=\tau \frac{\cos \theta_{\mathrm{Z}}}{\cos \theta_{\mathrm{W}}} \frac{M_{W}}{M_{Z^{\prime}}}
$$

Next, we can substitute the relations found in Eq. (2.20) into Eqs. (2.23) and (2.24) together with the definition of the right-handed couplings defined in Eq. (2.7), resulting in

$$
M_{Z}^{2}=v^{2} e^{2}\left(C_{Z \nu \nu}^{L}-C_{Z \nu \nu}^{R}\right)^{2}+w^{2} g_{z}^{\prime 2} \sin ^{2} \theta_{Z}
$$

and also using Eq. (2.8),

$$
M_{Z^{\prime}}^{2}=v^{2} e^{2}\left(C_{Z^{\prime} \nu \nu}^{L}-C_{Z^{\prime} \nu \nu}^{R}\right)^{2}+w^{2} g_{z}^{\prime 2} \cos ^{2} \theta_{Z}
$$

From Eqs. (2.25) and (2.26), we can express

$w g_{z}^{\prime} \sin \theta_{Z}=M_{Z} \sin \theta_{\mathrm{G}} \quad$ and $\quad w g_{z}^{\prime} \cos \theta_{Z}=M_{Z^{\prime}} \cos \theta_{\mathrm{G}}$,

which after substitution and simple rearrangement leads to

$$
\begin{aligned}
M_{Z}^{2} & =\frac{v^{2} e^{2}}{\cos ^{2} \theta_{\mathrm{G}}}\left(C_{Z \nu \nu}^{L}-C_{Z \nu \nu}^{R}\right)^{2}, \\
M_{Z^{\prime}}^{2} & =\frac{v^{2} e^{2}}{\sin ^{2} \theta_{\mathrm{G}}}\left(C_{Z^{\prime} \nu \nu}^{L}-C_{Z^{\prime} \nu \nu}^{R}\right)^{2} .
\end{aligned}
$$

\section{Mass terms and mixing of neutrinos}

The masses of the neutrinos are generated by the leptonic Yukawa terms in the Lagrangian [30],

$-\mathcal{L}_{Y}^{\ell}=\frac{1}{2} \overline{\nu_{R}} \mathbf{Y}_{N}\left(\nu_{R}\right)^{c} \chi+\overline{\nu_{R}} \mathbf{Y}_{\nu} \varepsilon_{a b} L_{L a} \phi_{b}+$ H.c.

where $L_{L}$ is the left-handed lepton doublet, $\varepsilon_{a b}$ is the Levi-Civita symbol, $a$ and $b$ are $\mathrm{SU}(2)_{L}$ indices, $\mathbf{Y}_{N}$ and $\mathbf{Y}_{\nu}$ are $3 \times 3$ matrices, and the superscript $c$ denotes charge conjugation, $\nu^{c}=\mathrm{i} \gamma_{2} \nu^{*}$. After SSB, this Lagrangian becomes

$$
\begin{aligned}
-\mathcal{L}_{Y}^{\ell}= & \frac{w+s^{\prime}+\mathrm{i} \sigma_{\chi}}{2 \sqrt{2}} \overline{\nu_{R}} \mathbf{Y}_{N}\left(\nu_{R}\right)^{c} \\
& +\frac{v+h^{\prime}+\mathrm{i} \sigma_{\phi}}{\sqrt{2}} \overline{\nu_{R}} \mathbf{Y}_{\nu} \nu_{L}+\text { H.c. }
\end{aligned}
$$

and the terms proportional to the VEVs provide the mass matrices

$$
\mathbf{M}_{N}=\frac{w}{\sqrt{2}} \mathbf{Y}_{N}, \quad \mathbf{M}_{D}=\frac{v}{\sqrt{2}} \mathbf{Y}_{\nu}
$$

where we chose a basis such that the Majorana mass matrix $\mathbf{M}_{N}$ is real, positive, and diagonal, while the Dirac mass matrix $\mathbf{M}_{D}$ is complex.

In the flavor basis, the $6 \times 6$ mass matrix for the neutrinos can be written in terms of $3 \times 3$ blocks as

$$
\mathbf{M}^{\prime}=\left(\begin{array}{cc}
\mathbf{0}_{3} & \mathbf{M}_{D}^{T} \\
\mathbf{M}_{D} & \mathbf{M}_{N}
\end{array}\right)
$$

The weak (flavor) eigenstates $\left(\nu_{e}, \nu_{\mu}, \nu_{\tau}, \nu_{R, 1}^{c}, \nu_{R, 2}^{c}, \nu_{R, 3}^{c}\right)$ can be transformed into the basis of $\nu_{i}(i=1-6)$ mass eigenstates with a $6 \times 6$ unitary matrix [39] $\mathbf{U}$ where the mass matrix is diagonal,

$$
\mathbf{U}^{T} \mathbf{M}^{\prime} \mathbf{U}=\mathbf{M}=\operatorname{diag}\left(m_{1}, m_{2}, m_{3}, m_{4}, m_{5}, m_{6}\right) .
$$

It is helpful to decompose the matrix $\mathbf{U}$ into two $3 \times 6$ blocks $\mathbf{U}_{L}$ and $\mathbf{U}_{R}^{*}$,

$$
\mathbf{U}=\left(\begin{array}{c}
\mathbf{U}_{L} \\
\mathbf{U}_{R}^{*}
\end{array}\right)
$$

so $\mathbf{U}^{T}=\left(\mathbf{U}_{L}^{T}, \mathbf{U}_{R}^{\dagger}\right)$ where both blocks are $6 \times 3$ matrices. It may be worth emphasizing that despite what might be implied by the notation, the matrices $\mathbf{U}_{L}$ and $\mathbf{U}_{R}^{*}$ are only semiunitary. Useful relations of these matrices are collected in Appendix A.

\section{E. Gauge boson-neutrino interactions}

As the neutral currents are written in terms of flavor eigenstates, the interactions between the neutral gauge bosons and the propagating mass eigenstate neutrinos include also the neutrino mixing matrices:

$$
\boldsymbol{\Gamma}_{V \nu_{i} \nu_{j}}^{\mu}=-\mathrm{i} e \gamma^{\mu}\left(\boldsymbol{\Gamma}_{V \nu \nu}^{L} P_{L}+\boldsymbol{\Gamma}_{V \nu \nu}^{R} P_{R}\right)_{i j},
$$

where

$$
\boldsymbol{\Gamma}_{V \nu \nu}^{L}=C_{V \nu \nu}^{L} \mathbf{U}_{L}^{\dagger} \mathbf{U}_{L}-C_{V \nu \nu}^{R} \mathbf{U}_{R}^{T} \mathbf{U}_{R}^{*}
$$

and

$$
\boldsymbol{\Gamma}_{V \nu \nu}^{R}=-C_{V \nu \nu}^{L} \mathbf{U}_{L}^{T} \mathbf{U}_{L}^{*}+C_{V \nu \nu}^{R} \mathbf{U}_{R}^{\dagger} \mathbf{U}_{R}=-\left(\boldsymbol{\Gamma}_{V \nu \nu}^{L}\right)^{*}
$$

for both $V=Z$ and $V=Z^{\prime}$.

\section{F. Scalar boson-neutrino and Goldstone boson-neutrino interactions}

The terms containing the scalar and Goldstone bosons in Eq. (2.32) provide interactions between those and the neutrinos. These interactions have the same structure with small differences. For the propagating scalar states $S_{k}$ or $\sigma_{k}$ ( $k=1$ denoting $h$ or the Goldstone boson belonging to $Z$ and $k=2$ referring to $s$ or the Goldstone boson belonging to $Z^{\prime}$ ), such interactions can be decomposed into left- and right-chiral terms

$$
\boldsymbol{\Gamma}_{S_{k} / \sigma_{k} \nu_{i} \nu_{j}}=\left(\boldsymbol{\Gamma}_{S_{k} / \sigma_{k} \nu \nu}^{L} P_{L}+\boldsymbol{\Gamma}_{S_{k} / \sigma_{k} \nu \nu}^{R} P_{R}\right)_{i j},
$$


where the matrices $\boldsymbol{\Gamma}^{L / R}$ contain both the mixing matrix of the neutrinos and the mixing matrix of the scalar or Goldstone bosons. The left-chiral coefficients are

$$
\begin{aligned}
\boldsymbol{\Gamma}_{S_{k} \nu \nu}^{L}= & -\mathrm{i}\left[\left(\mathbf{M} \mathbf{U}_{L}^{\dagger} \mathbf{U}_{L}+\mathbf{U}_{L}^{T} \mathbf{U}_{L}^{*} \mathbf{M}\right) \frac{\left(\mathbf{Z}_{S}\right)_{k 1}}{v}\right. \\
& \left.+\mathbf{U}_{R}^{\dagger} \mathbf{M}_{N} \mathbf{U}_{R}^{*} \frac{\left(\mathbf{Z}_{S}\right)_{k 2}}{w}\right]
\end{aligned}
$$

and

$$
\begin{aligned}
\boldsymbol{\Gamma}_{\sigma_{k} \nu \nu}^{L}= & -\left[\left(\mathbf{M} \mathbf{U}_{L}^{\dagger} \mathbf{U}_{L}+\mathbf{U}_{L}^{T} \mathbf{U}_{L}^{*} \mathbf{M}\right) \frac{\left(\mathbf{Z}_{\mathrm{G}}\right)_{k 1}}{v}\right. \\
& \left.+\mathbf{U}_{R}^{\dagger} \mathbf{M}_{N} \mathbf{U}_{R}^{*} \frac{\left(\mathbf{Z}_{\mathrm{G}}\right)_{k 2}}{w}\right]
\end{aligned}
$$

and the right-chiral ones are related by complex conjugation, $\boldsymbol{\Gamma}_{S_{k} / \sigma_{k} \nu \nu}^{R}=-\left(\boldsymbol{\Gamma}_{S_{k} / \sigma_{k} \nu \nu}^{L}\right)^{*}$.

\section{NEUTRINO MASS MATRIX AT ONE-LOOP ORDER}

We are interested in the one-loop correction $\delta \mathbf{M}_{L}$ to the tree-level mass matrix of the light neutrinos. In perturbation theory, we deal with propagating states which are mass eigenstates. Hence, we can compute loop corrections to self-energies of mass eigenstates of neutrinos. The neutrino mass matrix at one-loop order is then obtained from Eq. (2.35), with diagonal mass matrix substituted at one loop, $\mathbf{M}+\delta \mathbf{M}$ where

$$
\delta \mathbf{M}=\operatorname{diag}\left(\delta m_{1}, \delta m_{2}, \delta m_{3}, \delta m_{4}, \delta m_{5}, \delta m_{6}\right) .
$$

Hence, the correction is obtained by

$$
\delta \mathbf{M}^{\prime}=\left(\begin{array}{ll}
\delta \mathbf{M}_{L} & \delta \mathbf{M}_{D}^{T} \\
\delta \mathbf{M}_{D} & \delta \mathbf{M}_{N}
\end{array}\right)=\mathbf{U}^{*} \delta \mathbf{M U}^{\dagger} .
$$

Using Eq. (2.36), we can compute the $3 \times 3$ blocks as

$$
\begin{aligned}
\delta \mathbf{M}_{L} & =\mathbf{U}_{L}^{*} \delta \mathbf{M} \mathbf{U}_{L}^{\dagger}, \quad \delta \mathbf{M}_{D}=\mathbf{U}_{R} \delta \mathbf{M} \mathbf{U}_{L}^{\dagger}, \\
\delta \mathbf{M}_{N} & =\mathbf{U}_{R} \delta \mathbf{M} \mathbf{U}_{R}^{T} .
\end{aligned}
$$

In the following subsections, we prove that the one-loop correction to the mass matrix of the active neutrinos has the form

$$
\begin{aligned}
\delta \mathbf{M}_{L}= & \frac{1}{16 \pi^{2}} \sum_{k=1,2}\left[3\left(\mathbf{Z}_{\mathrm{G}}\right)_{k 1}^{2} \frac{M_{V_{k}}^{2}}{v^{2}} \mathbf{F}\left(M_{V_{k}}^{2}\right)\right. \\
& \left.+\left(\mathbf{Z}_{\mathrm{S}}\right)_{k 1}^{2} \frac{M_{S_{k}}^{2}}{v^{2}} \mathbf{F}\left(M_{S_{k}}^{2}\right)\right]
\end{aligned}
$$

where we introduced the finite matrix valued function

$$
\mathbf{F}_{i j}\left(M^{2}\right)=\sum_{a=1}^{6}\left(\mathbf{U}_{L}^{*}\right)_{i a}\left(\mathbf{U}_{L}^{\dagger}\right)_{a j} \frac{m_{a}^{3}}{M^{2}} \frac{\ln \frac{m_{a}^{2}}{M^{2}}}{\frac{m_{a}^{2}}{M^{2}}-1}
$$

of dimension mass and with summation running over all neutrinos.

\section{A. Self-energy decomposition}

The neutrino self-energy is a $6 \times 6$ matrix that can be decomposed as

$$
\begin{aligned}
\mathrm{i} \boldsymbol{\Sigma}(p)= & \mathbf{A}_{L}\left(p^{2}\right) \not p P_{L}+\mathbf{A}_{R}\left(p^{2}\right) \not p P_{R}+\mathbf{B}_{L}\left(p^{2}\right) P_{L} \\
& +\mathbf{B}_{R}\left(p^{2}\right) P_{R} .
\end{aligned}
$$

Using this decomposition, $\delta \mathbf{M}_{L}$ is given by [26]

$$
\delta \mathbf{M}_{L}=\mathbf{U}_{L}^{*} \mathbf{B}_{L}(0) \mathbf{U}_{L}^{\dagger} .
$$

The matrix $\mathbf{B}_{L}(0)$ receives contributions involving a neutrino and either a neutral vector boson Z, Z', or a scalar boson $\sigma_{Z}, \sigma_{Z^{\prime}}$ (Goldstone boson), $h, s$ (Higgs-like scalar) in the loop. The relevant Feynman graphs that give contributions to the neutrino self-energies at one-loop order are shown in Fig. 1. There are also tadpole contributions to $\mathbf{B}_{L}(0)$. Those are proportional to the scalar-neutrino coupling $\Gamma_{S_{k} \nu_{i} \nu_{j}}^{L}$ given in Eq. (2.40), which vanishes when sandwiched between $\mathbf{U}_{L}^{*}$ and $\mathbf{U}_{L}^{\dagger}$; see Eq. (A5). The charged vector boson together with a charged lepton in the loop (bottom right diagram in Fig. 1) contributes only to $\mathbf{A}_{L / R}$. Thus, we compute the first three graphs explicitly. For a given boson $x$ in the loop, the matrix $\mathbf{B}_{L}(0)$ depends on the mass $M_{x}$ and also the tree-level masses of the neutrinos $\left\{m_{a}\right\}, \mathbf{B}_{L}(0)=\mathbf{B}_{L}^{x}\left(M_{x},\left\{m_{a}\right\}\right)$.

\section{B. Contributions with neutral gauge bosons in the loop}

The contribution of the neutral gauge boson $V$ is ${ }^{1}$

$$
\begin{aligned}
& \left(B_{L}^{V}\left(M_{V},\left\{m_{a}\right\} ; \xi_{V}\right)\right)_{i j} P_{L} \\
& =\mathrm{i} \int \frac{\mathrm{d}^{d} \ell}{(2 \pi)^{d}} \sum_{a=1}^{6} \Gamma_{V \nu_{i} \nu_{a}}^{\mu} \frac{\not p-\not \ell+m_{a}}{(p-\ell)^{2}-m_{a}^{2}} \Gamma_{V \nu_{a} \nu_{j}}^{\nu} P_{\mu \nu}\left(\ell, M_{V}^{2} ; \xi_{V}\right),
\end{aligned}
$$

where $\xi_{V}$ is the gauge parameter and

$$
\begin{aligned}
& P_{\mu \nu}\left(\ell, M_{V}^{2} ; \xi_{V}\right) \\
& \quad=\frac{g_{\mu \nu}}{\ell^{2}-M_{V}^{2}}-\left(1-\xi_{V}\right) \frac{\ell_{\mu} \ell_{\nu}}{\left(\ell^{2}-M_{V}^{2}\right)\left(\ell^{2}-\xi_{V} M_{V}^{2}\right)} .
\end{aligned}
$$

Introducing the $6 \times 6$ matrix

\footnotetext{
${ }^{1}$ We suppress $\mu^{2 \varepsilon}$ in the following integrals until Eq. (3.18) where it is shown explicitly. $\mu$ is the mass parameter of dimensional regularization.
} 


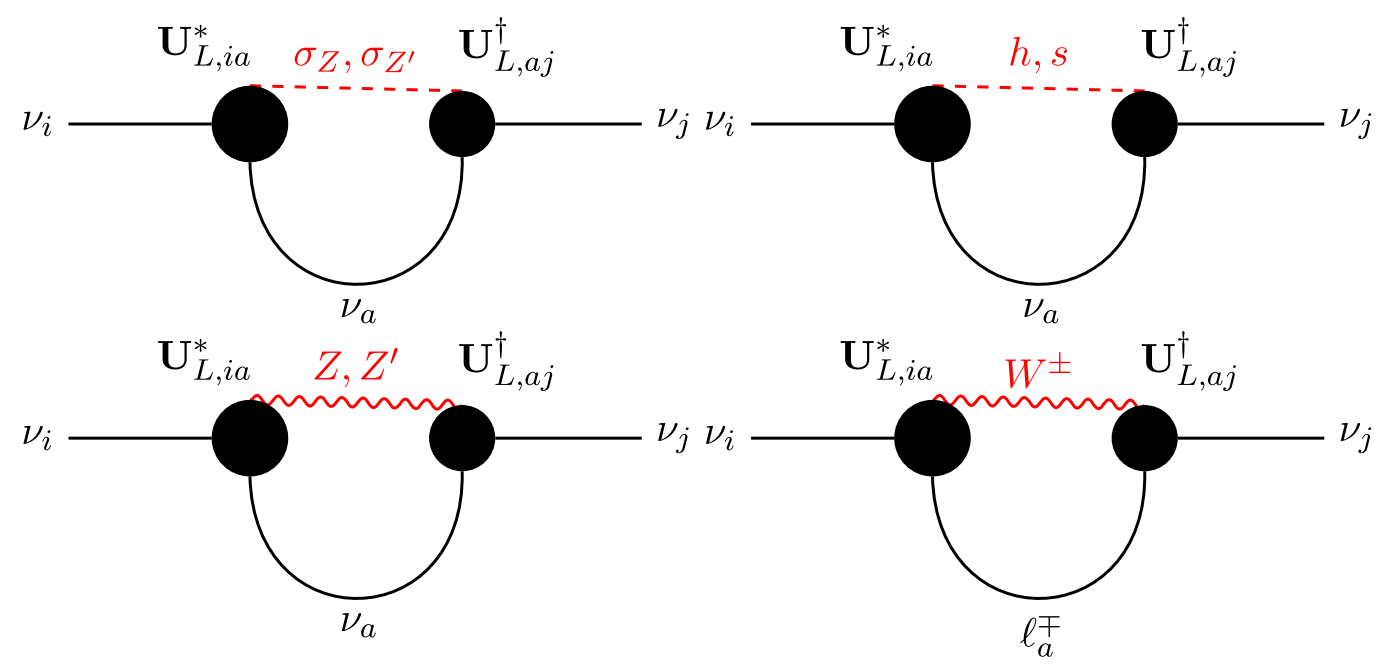

FIG. 1. One-loop graphs contributing to the neutrino self-energy. Top left: Goldstone boson contribution. Top right: scalar contribution. Bottom left: neutral gauge boson contribution. Bottom right: charged gauge boson contribution. Note that the $W$ boson loop does not contribute to the matrix $\mathbf{B}_{L}$.

$$
\mathbf{m}_{\ell}^{(n)}=\operatorname{diag}\left(\frac{m_{1}^{n}}{\ell^{2}-m_{1}^{2}}, \ldots, \frac{m_{6}^{n}}{\ell^{2}-m_{6}^{2}}\right),
$$

and using the result of Appendix B, we obtain the following expression for a neutral vector boson in the loop:

$$
\begin{aligned}
\delta \mathbf{M}_{L}^{V}= & \mathrm{i} e^{2}\left(C_{V \nu \nu}^{L}-C_{V \nu \nu}^{R}\right)^{2} \int \frac{\mathrm{d}^{d} \ell}{(2 \pi)^{d}} \\
& \times \mathbf{U}_{L}^{*}\left[\frac{d \mathbf{m}_{\ell}^{(1)}}{\ell^{2}-M_{V}^{2}}+\frac{\mathbf{m}_{\ell}^{(3)}}{M_{V}^{2}}\left(\frac{1}{\ell^{2}-\xi_{V} M_{V}^{2}}-\frac{1}{\ell^{2}-M_{V}^{2}}\right)\right] \mathbf{U}_{L}^{\dagger} .
\end{aligned}
$$

\section{Contributions with neutral Goldstone bosons in the loop}

The contribution of the neutral Goldstone boson $\sigma_{V}$ ( $V=1$ means the Goldstone boson belonging to the $Z$ field and $V=2$ refers to the $Z^{\prime}$ field) is

$$
\begin{aligned}
& \left(B_{L}^{\sigma_{V}}\left(m_{\sigma_{V}},\left\{m_{a}\right\} ; \xi_{V}\right)\right)_{i j} P_{L} \\
& \quad=-\mathrm{i} \int \frac{\mathrm{d}^{d} \ell}{(2 \pi)^{d}} \sum_{a=1}^{6} \boldsymbol{\Gamma}_{\sigma_{V} \nu_{i} \nu_{a}} \frac{m_{a}}{\ell^{2}-m_{a}^{2}} \boldsymbol{\Gamma}_{\sigma_{V} \nu_{a} \nu_{j}} \frac{1}{\ell^{2}-\xi_{V} M_{V}^{2}} .
\end{aligned}
$$

Using the matrix notation, we can write

$$
\begin{aligned}
& \mathbf{U}_{L}^{*} \mathbf{B}_{L}^{\sigma_{V}}(0) \mathbf{U}_{L}^{\dagger} P_{L} \\
& \quad=-\mathrm{i} \int \frac{\mathrm{d}^{d} \ell}{(2 \pi)^{d}} \mathbf{U}_{L}^{*} \boldsymbol{\Gamma}_{\sigma_{V} \nu} \mathbf{m}_{\ell}^{(1)} \boldsymbol{\Gamma}_{\sigma_{V} \nu \nu} \mathbf{U}_{L}^{\dagger} \frac{1}{\ell^{2}-\xi_{V} M_{V}^{2}} .
\end{aligned}
$$

Substituting the vertex functions of Eq. (2.40) and employing the matrix relations in Eqs. (A2) and (A5), we obtain the correction to the mass matrix as

$$
\delta \mathbf{M}_{L}^{\sigma_{V}}=-\mathrm{i} \int \frac{\mathrm{d}^{d} \ell}{(2 \pi)^{d}} \mathbf{U}_{L}^{*} \mathbf{M} \mathbf{m}_{\ell}^{(1)} \mathbf{M} \mathbf{U}_{L}^{\dagger}\left(\frac{\left(\mathbf{Z}_{\mathrm{G}}\right)_{V 1}}{v}\right)^{2} \frac{1}{\ell^{2}-\xi_{V} M_{V}^{2}} .
$$

We now substitute $\mathbf{M m}_{\ell}^{(1)} \mathbf{M}=\mathbf{m}_{\ell}^{(3)}$ and using Eq. (2.30), we obtain

$$
\delta \mathbf{M}_{L}^{\sigma_{V}}=-\mathrm{i} e^{2}\left(C_{V \nu \nu}^{L}-C_{V \nu \nu}^{R}\right)^{2} \int \frac{\mathrm{d}^{d} \ell}{(2 \pi)^{d}} \mathbf{U}_{L}^{*} \frac{\mathbf{m}_{\ell}^{(3)}}{M_{V}^{2}} \mathbf{U}_{L}^{\dagger} \frac{1}{\ell^{2}-\xi_{V} M_{V}^{2}}
$$

\section{Contributions with scalar bosons in the loop}

The scalar-neutrino vertex is very similar to the Goldstone boson neutrino vertex, so the contribution with a scalar boson $S_{k}$ in the loop can be written immediately in analogy with Eq. (3.14):

$$
\begin{aligned}
\delta \mathbf{M}_{L}^{S_{k}} & =\mathrm{i} \int \frac{\mathrm{d}^{d} \ell}{(2 \pi)^{d}} \mathbf{U}_{L}^{*} \mathbf{M} \mathbf{m}_{\ell}^{(1)} \mathbf{M} \mathbf{U}_{L}^{\dagger}\left(\frac{\left(\mathbf{Z}_{S}\right)_{k 1}}{v}\right)^{2} \frac{1}{\ell^{2}-M_{S_{k}}^{2}} \\
& =\mathrm{i}\left(\frac{\left(\mathbf{Z}_{S}\right)_{k 1}}{v}\right)^{2} \int \frac{\mathrm{d}^{d} \ell}{(2 \pi)^{d}} \mathbf{U}_{L}^{*} \mathbf{m}_{\ell}^{(3)} \mathbf{U}_{L}^{\dagger} \frac{1}{\ell^{2}-M_{S_{k}}^{2}} .
\end{aligned}
$$

\section{E. The complete one-loop mass correction}

Combining Eqs. (3.11), (3.15), and (3.16), we find that that the gauge-dependent pieces of the vector boson contribution cancel exactly with the Goldstone boson contribution, and obtain 


$$
\begin{aligned}
\delta \mathbf{M}_{L}= & \sum_{V=Z, Z^{\prime}}\left(\delta \mathbf{M}_{L}^{V}+\delta \mathbf{M}_{L}^{\sigma_{V}}\right)+\sum_{k=1,2} \delta \mathbf{M}_{L}^{S_{k}} \\
= & \sum_{V=Z, Z^{\prime}} \mathrm{i} e^{2}\left(C_{V \nu \nu}^{L}-C_{V \nu \nu}^{R}\right)^{2} \int \frac{\mathrm{d}^{d} \ell}{(2 \pi)^{d}} \\
& \times \mathbf{U}_{L}^{*}\left[\frac{d \mathbf{m}_{\ell}^{(1)}}{\ell^{2}-M_{V}^{2}}-\frac{\mathbf{m}_{\ell}^{(3)}}{M_{V}^{2}} \frac{1}{\ell^{2}-M_{V}^{2}}\right] \mathbf{U}_{L}^{\dagger} \\
& +\sum_{k=1,2} \mathrm{i}\left(\frac{\left(\mathbf{Z}_{S}\right)_{k 1}}{v}\right)^{2} \int \frac{\mathrm{d}^{d} \ell}{(2 \pi)^{d}} \mathbf{U}_{L}^{*} \frac{\mathbf{m}_{\ell}^{(3)}}{\ell^{2}-M_{S_{k}}^{2}} \mathbf{U}_{L}^{\dagger} .
\end{aligned}
$$

Introducing the integral

$$
\begin{aligned}
& I_{0}\left(M^{2}, m_{a}^{2} ; \mu^{2}, \epsilon\right) \\
& \quad=\mu^{2 \epsilon} \int \frac{\mathrm{d}^{d} \ell}{(2 \pi)^{d}} \frac{1}{\left(\ell^{2}-M^{2}\right)\left(\ell^{2}-m_{a}^{2}\right)},
\end{aligned}
$$

the matrix $\mathbf{I}^{(n)}$ with elements

$\mathbf{I}_{i j}^{(n)}\left(M^{2}\right)=\mathrm{i} \sum_{a=1}^{6}\left(\mathbf{U}_{L}^{*}\right)_{i a} m_{a}^{n}\left(\mathbf{U}_{L}^{\dagger}\right)_{a j} I_{0}\left(M^{2}, m_{a}^{2} ; \mu^{2}, \epsilon\right)$,

and using the relations (2.30) allows us to recast Eq. (3.17) into a neatly condensed form

$$
\begin{aligned}
\delta \mathbf{M}_{L}= & \sum_{k=1,2}\left\{\left(\frac{\left(\mathbf{Z}_{\mathrm{G}}\right)_{k 1}}{v}\right)^{2}\left(d M_{V_{k}}^{2} \mathbf{I}^{(1)}\left(M_{V_{k}}^{2}\right)-\mathbf{I}^{(3)}\left(M_{V_{k}}^{2}\right)\right)\right. \\
& \left.+\left(\frac{\left(\mathbf{Z}_{S}\right)_{k 1}}{v}\right)^{2} \mathbf{I}^{(3)}\left(M_{S_{k}}^{2}\right)\right\}
\end{aligned}
$$

with $V_{1}=Z$ and $V_{2}=Z^{\prime}$. In Eq. (3.18), $2 \epsilon=4-d$ and $\mu$ is the regularization scale.

\section{F. Finiteness and scale independence of $\delta \mathrm{M}_{L}$}

We show here that the one-loop mass correction $\delta \mathbf{M}_{L}$ is finite and independent of the scale $\mu$. Evaluating the integral (3.18) yields

$I_{0}\left(M^{2}, m^{2} ; \mu^{2}, \epsilon\right)=I_{0}^{(\mathrm{s})}(\epsilon)+I_{0}^{(\mathrm{f})}\left(\frac{m^{2}}{M^{2}}, \frac{\mu^{2}}{M^{2}}\right)+\mathrm{O}(\epsilon)$,

where "s" stands for the singular and " $\mathrm{f}$ " for the finite functions

$$
\begin{aligned}
I_{0}^{(\mathrm{s})}(\epsilon) & =\frac{\mathrm{i}}{16 \pi^{2}}\left(\frac{1}{\epsilon}-\gamma_{\mathrm{E}}+\ln 4 \pi+1\right), \\
I_{0}^{(\mathrm{f})}\left(x, x_{\mu}\right) & =\frac{\mathrm{i}}{16 \pi^{2}}\left(\frac{x \ln x}{1-x}+\ln x_{\mu}\right)
\end{aligned}
$$

with $\gamma_{\mathrm{E}} \simeq 0.5722$ being the Euler-Mascheroni constant. It is also convenient to split the matrix (3.19) in a similar fashion

$$
\mathbf{I}^{(n)}\left(M^{2}\right)=\mathbf{I}^{(\mathrm{s}, n)}+\mathbf{I}^{(\mathrm{f}, n)}\left(M^{2}\right),
$$

such that

$$
\begin{aligned}
\mathbf{I}^{(\mathrm{s}, n)} & =\mathrm{i} \mathbf{U}_{L}^{*} \mathbf{M}^{n} \mathbf{U}_{L}^{\dagger} I_{0}^{(\mathrm{s}, n)}, \\
\left(\mathbf{I}^{(\mathrm{f}, n)}\left(M^{2}\right)\right)_{i j} & =\mathrm{i} \sum_{a=1}^{6}\left(\mathbf{U}_{L}^{*}\right)_{i a} m_{a}^{n}\left(\mathbf{U}_{L}^{\dagger}\right)_{a j} I_{0}^{(\mathrm{f}, n)}\left(\frac{m_{a}^{2}}{M^{2}}, \frac{\mu^{2}}{M^{2}}\right) .
\end{aligned}
$$

Then the one-loop correction to the mass matrix of the light neutrinos can also be decomposed as

$$
\delta \mathbf{M}_{L}=\delta \mathbf{M}_{L}^{(\mathrm{s})}+\delta \mathbf{M}_{L}^{(\mathrm{f})}+\mathrm{O}(\epsilon)
$$

where

$$
\begin{aligned}
\delta \mathbf{M}_{L}^{(\mathrm{s})}= & \sum_{k=1,2}\left[d M_{V_{k}}^{2}\left(\frac{\left(\mathbf{Z}_{\mathrm{G}}\right)_{k 1}}{v}\right)^{2} \mathbf{I}^{(\mathrm{s}, 1)}\right. \\
& \left.+\frac{\left(\mathbf{Z}_{S}\right)_{k 1}^{2}-\left(\mathbf{Z}_{\mathrm{G}}\right)_{k 1}^{2}}{v^{2}} \mathbf{I}^{(\mathrm{s}, 3)}\right]
\end{aligned}
$$

and

$$
\begin{aligned}
\delta \mathbf{M}_{L}^{(\mathrm{f})}= & \sum_{k=1,2}\left[\left(\frac{\left(\mathbf{Z}_{\mathrm{G}}\right)_{k 1}}{v}\right)^{2}\left(d M_{V_{k}}^{2} \mathbf{I}^{(\mathrm{f}, 1)}\left(M_{V_{k}}^{2}\right)-\mathbf{I}^{(\mathrm{f}, 3)}\left(M_{V_{k}}^{2}\right)\right)\right. \\
& \left.+\left(\frac{\left(\mathbf{Z}_{S}\right)_{k 1}}{v}\right)^{2} \mathbf{I}^{(\mathrm{f}, 3)}\left(M_{S_{k}}^{2}\right)\right]
\end{aligned}
$$

In order to prove that $\delta \mathbf{M}_{L}$ is finite, one has to show that $\delta \mathbf{M}_{L}^{(\mathrm{s})}$ is free from $\epsilon$ poles. We prove that it in fact vanishes because the matrix $\mathbf{I}^{(\mathrm{s}, 1)}$ is a zero matrix due to the identity (A5), while the coefficient in the second term cancels because the matrices $\mathbf{Z}_{S}$ and $\mathbf{Z}_{G}$ are orthogonal, so

$$
\sum_{k=1}^{2}\left(\mathbf{Z}_{S}\right)_{k 1}^{2}-\sum_{k=1}^{2}\left(\mathbf{Z}_{G}\right)_{k 1}^{2}=0 .
$$

Hence, the mass-independent terms, including the divergent pieces of the light neutrino one-loop mass correction cancel, and we can set $\epsilon=0$, which yields $\delta \mathbf{M}_{L}=\delta \mathbf{M}_{L}^{(\mathrm{f})}$. Furthermore, the terms depending on the regularization scale in $\delta \mathbf{M}_{L}^{(\mathrm{f})}$ cancel in an identical way as the second term does in $\delta \mathbf{M}_{L}^{(\mathrm{s})}$ [using Eq. (3.28)].

The remaining finite terms give the final, regularizationscale-independent and finite one-loop correction to the light neutrino mass matrix as given in Eq. (3.4). It is the linear combination of the matrix valued function $\mathbf{F}$ given in Eq. (3.5) with different arguments and coefficients corresponding to the different one-loop contributions. The function $\mathbf{F}$ gives the mass correction corresponding to a 
one-loop diagram before coupling suppression; see Fig. 4 in Sec. IV for details where we shall also give a numerical estimate for its eigenvalues $\delta m_{i, 0}^{\nu}$. It is well defined for any non-negative $x$ because

$$
\lim _{x \rightarrow 0} \frac{x \ln x}{1-x}=0 \quad \text { and } \quad \lim _{x \rightarrow 1} \frac{x \ln x}{1-x}=-1 .
$$

\section{G. Generalization to arbitrary number of neutral bosons and neutrinos}

Our predictions for the one-loop correction to the light neutrino mass matrix can easily be generalized to any number of $n_{V}$ massive neutral gauge bosons originating from an extension by a $U(1)^{n_{V}}$ Abelian gauge group, $n_{S}$ neutral real scalars coupling to $n_{a}$ active and $n_{s}$ sterile neutrinos. Clearly, the matrix form of gauge-dependent parts in Eqs. (3.11) and (3.15) is unchanged, and they cancel in the same way.

The correction without gauge parameters $\xi_{V}$ in Eq. (3.4) is straightforwardly generalized to a case where the sums go over an arbitrary positive integer $n_{V}$ and $n_{S}$.
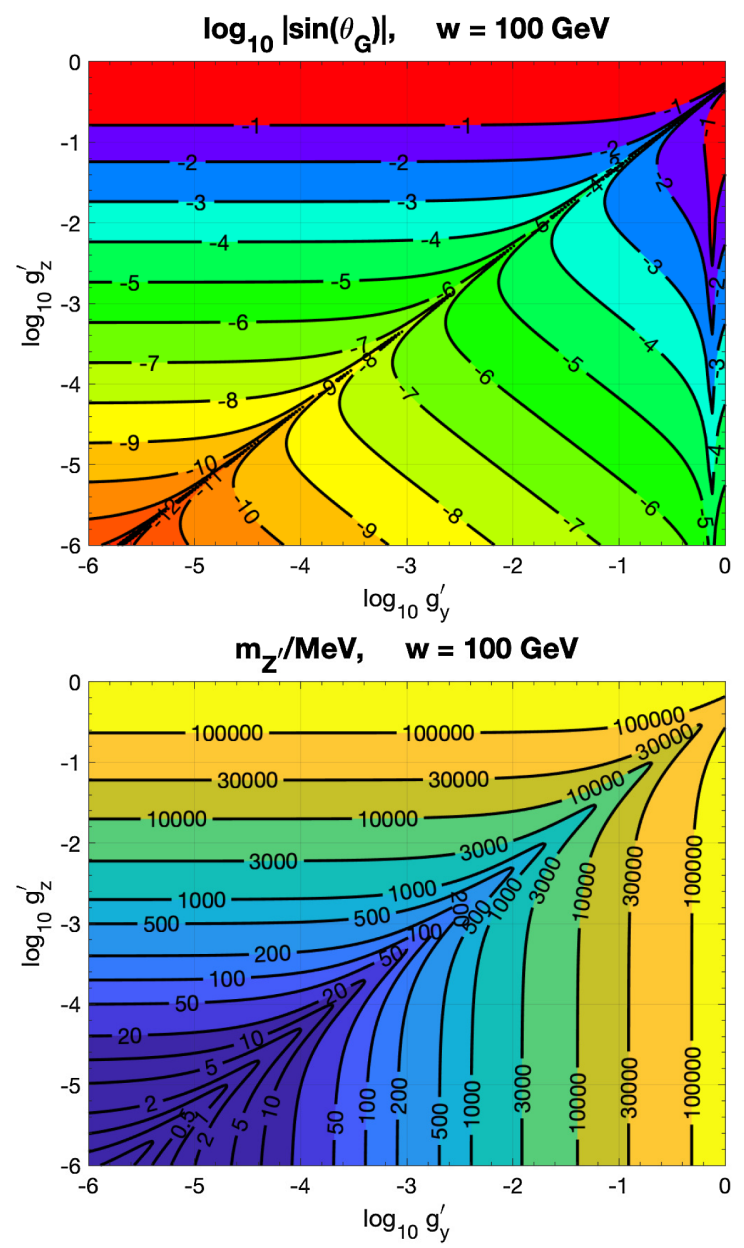

The neutrino mass and mixing matrices with arbitrary $n_{a}$ and $n_{s}$ are written identically in the block form, differing only on the block shape: $\mathbf{U}_{L}$ is an $n_{a} \times\left(n_{a}+n_{s}\right)$ matrix and $\mathbf{U}_{R}$ is an $n_{s} \times\left(n_{a}+n_{s}\right)$ matrix. The finite correction presented in Eq. (3.4) is then immediately generalized to

$$
\begin{aligned}
\delta \mathbf{M}_{L}= & \frac{1}{16 \pi^{2}}\left[3 \sum_{k=1}^{n_{V}}\left(\mathbf{Z}_{\mathrm{G}}\right)_{k 1}^{2} \frac{M_{V_{k}}^{2}}{v^{2}} \mathbf{F}\left(M_{V_{k}}^{2}\right)\right. \\
& \left.+\sum_{k=1}^{n_{S}}\left(\mathbf{Z}_{\mathrm{S}}\right)_{k 1}^{2} \frac{M_{S_{k}}^{2}}{v^{2}} \mathbf{F}\left(M_{S_{k}}^{2}\right)\right],
\end{aligned}
$$

where the upper limit in the summation in the matrix $\mathbf{F}$ is $n_{a}+n_{s}$. The factor 3 in front of the first term in the brackets of Eq. (3.30) stems from the three polarization states of the propagating massive neutral gauge bosons. The corresponding factor is, of course, one in the case of the scalars. This formula is also independent of the new $\mathrm{U}(1)$ charge assignments.
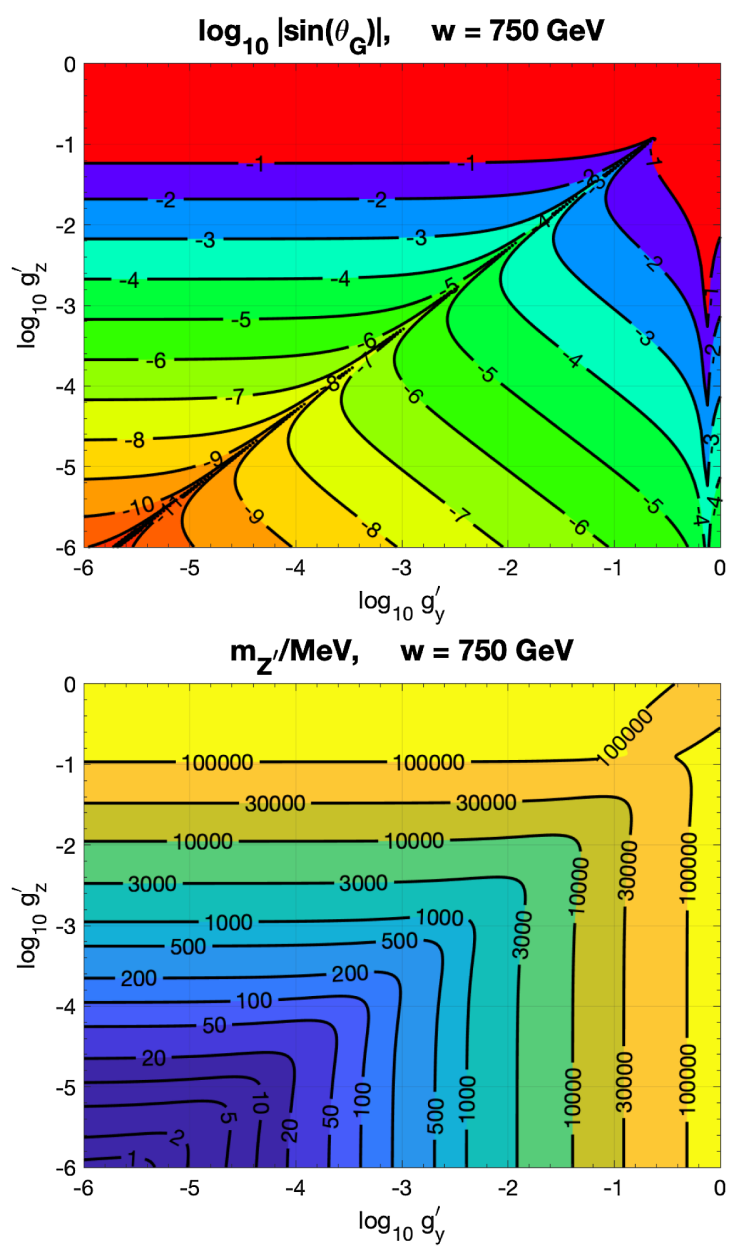

FIG. 2. Absolute values of $\sin \theta_{G}$ (top) and mass of $Z^{\prime}$ boson (bottom) in the logarithmic $\left(g_{y}^{\prime}, g_{z}^{\prime}\right)$ plane. For $\theta_{G}$ the contour labels $n$ correspond to value $10^{n}$. Left plots: $w=100 \mathrm{GeV}$. Right plots: $w=750 \mathrm{GeV}$. 

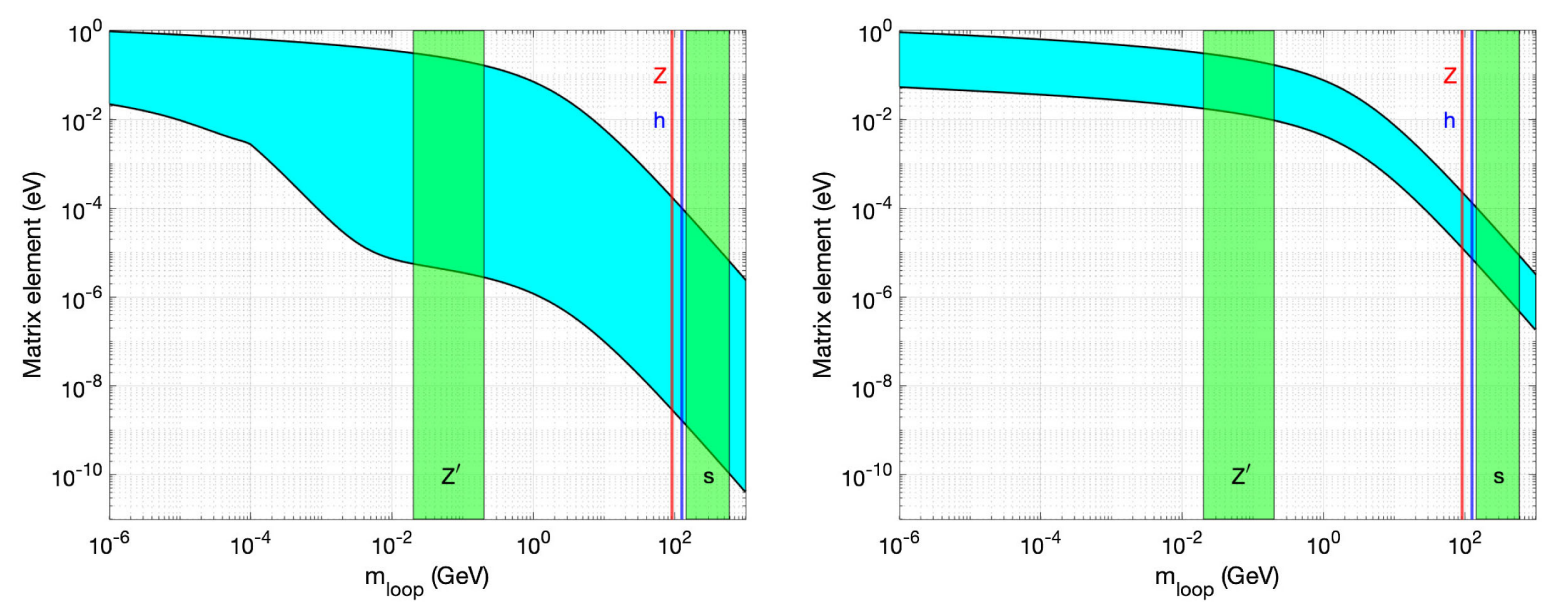

FIG. 3. Matrix elements $\mathbf{F}_{i j}$ as a function of the mass $m_{\text {loop }}$ of the boson in the loop are confined to the blue band, assuming normal neutrino mass hierarchy. We have highlighted with vertical bands the relevant mass regions where the masses of the bosons in the loop lie. The scalar $s$ is required to have mass between 144 and $558 \mathrm{GeV}$ requiring stability of the vacuum [40]. Left plot: $m_{1}^{\text {tree }}=0.01 \mathrm{eV}$, $m_{4}^{\text {tree }}=30 \mathrm{keV}, m_{5}^{\text {tree }} \approx m_{6}^{\text {tree }}=2.5 \mathrm{GeV}$. Right plot: $m_{1}^{\text {tree }}=0.001 \mathrm{eV}, m_{4}^{\text {tree }}=7.1 \mathrm{keV}, m_{5}^{\text {tree }} \approx m_{6}^{\text {tree }}=3.0 \mathrm{GeV}$.

\section{NUMERICAL ESTIMATE OF THE CORRECTIONS}

We are now ready to estimate the order of magnitude of the corrections. We assume large mixing in the scalar sector: $\theta_{S}=\mathrm{O}(1)$. The $Z^{\prime}$ mass and mixing angle $\theta_{G}$ are fixed by the gauge couplings $g_{y}^{\prime}=\gamma_{y}^{\prime} g_{L}$ and $g_{z}^{\prime}$ and ratio of VEVs, $\tan \beta \equiv w / v$. We plot their magnitudes in Fig. 2, scanning the parameters $g_{y}^{\prime}, g_{z}^{\prime} \in\left[10^{-6}, 1\right]$ and for $w=100$, $750 \mathrm{GeV}$. Note that larger $\tan \beta$ corresponds to a larger Goldstone angle. Smaller $\tan \beta$ distorts the $M_{Z^{\prime}}$ contours so that the same $Z^{\prime}$ mass can be achieved with larger gauge couplings $g_{y}^{\prime}$ and $g_{z}^{\prime}$ compared to large $\tan \beta$. In addition, we set $M_{s} / v=\mathrm{O}(1)$; that is, only the mass of the $Z^{\prime}$ boson is free, and may be far from electroweak scale. The relevant gauge couplings can then be estimated as from Fig. 2 after identifying the region in the $\left(g_{y}^{\prime}, g_{z}^{\prime}\right)$ plane corresponding to $M_{Z^{\prime}} \in[20,200] \mathrm{MeV}$, which is the relevant mass region for the superweak model to reproduce the dark matter relic density allowed by experimental constraints [32].

Then we identify the order-of-magnitude estimate for $\left|\sin \theta_{G}\right|$ by comparing the regions relevant to the mass range of $M_{Z^{\prime}}$. For $w=100 \mathrm{GeV}$, we have $\left|\sin \theta_{G}\right|<10^{-6}$, which we take as a conservative upper limit. Then the prefactors in the gauge boson contributions to $\delta \mathbf{M}_{L}$ are

$$
e^{2}\left(C_{Z \nu \nu}^{L}-C_{Z \nu \nu}^{R}\right)^{2}=\cos ^{2} \theta_{G} \frac{M_{Z}^{2}}{v^{2}} \sim \mathrm{O}\left(10^{-1}\right)
$$

and

$$
\begin{aligned}
e^{2}\left(C_{Z^{\prime} \nu \nu}^{L}-C_{Z^{\prime} \nu \nu}^{R}\right)^{2} & =\sin ^{2} \theta_{G} \frac{M_{Z^{\prime}}^{2}}{v^{2}} \\
& \sim \mathrm{O}\left(10^{-19}\right) \times\left(\frac{M_{Z^{\prime}}}{100 \mathrm{MeV}}\right)^{2} .
\end{aligned}
$$

Then the numerical estimate for the total correction in Eq. (3.4) can be written as

$$
\begin{aligned}
\left(\delta \mathbf{M}_{L}\right)_{i j}< & \mathrm{O}\left(10^{-7}\right) \mathrm{eV} \\
& +\mathrm{O}\left(10^{-21}\right) \times\left(\frac{M_{Z^{\prime}}}{100 \mathrm{MeV}}\right)^{2} \mathbf{F}_{i j}\left(M_{Z^{\prime}}^{2}\right) .
\end{aligned}
$$

The elements of the matrix $\mathbf{F}$ are plotted as a function of the mass of the boson of the loop $m_{\text {loop }}$ in Fig. 3 and the eigenvalues of the matrix corresponding to corrections to active neutrino species in Fig. 4. The eigenvalues of $\mathbf{F}$ themselves exceed the active neutrino tree-level masses, as the latter are at most about $\mathrm{O}(1) \mathrm{eV}$ for the $\mathrm{MeV}$-scale

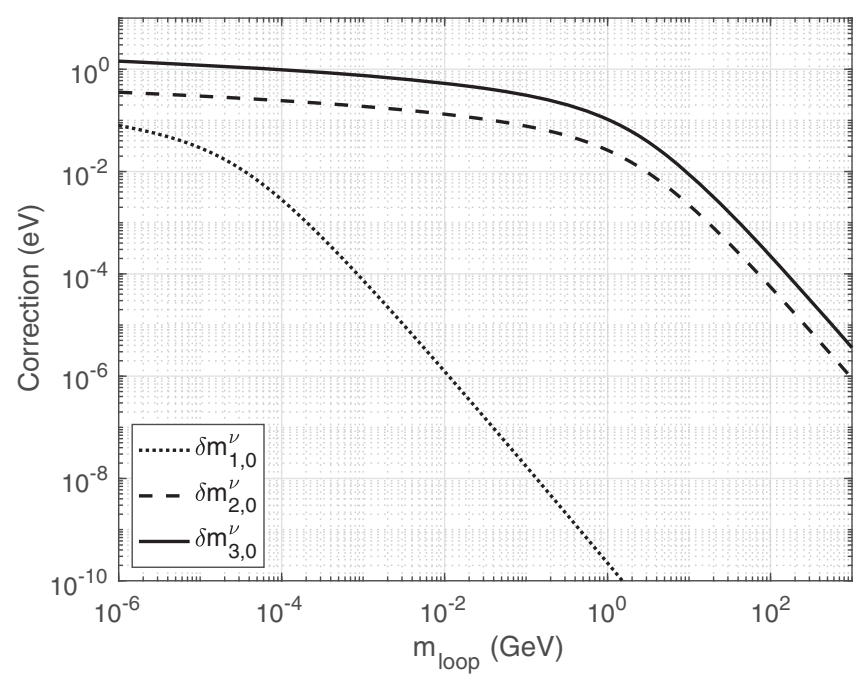

FIG. 4. Eigenvalues of the matrix $\mathbf{F}$ as a function of the mass of the boson in the loop $m_{\text {loop }}$, assuming $m_{1}^{\text {tree }}=0.01 \mathrm{eV}$, $m_{4}^{\text {tree }}=30 \mathrm{keV}, m_{5}^{\text {tree }} \approx m_{6}^{\text {tree }}=2.5 \mathrm{GeV}$, and normal neutrino mass hierarchy. 
$Z^{\prime}$ boson. However, the coupling suppressions in Eq. (4.3) are sufficient to tame the relative correction to the tree-level mass below the percent level. Assuming the active neutrino masses to be $\mathrm{O}\left(10^{-3}\right) \mathrm{eV}$, a rough estimate for the relative correction to active neutrino masses is of $\mathrm{O}\left(10^{-4}\right)$.

We may maximize the effect of the $Z^{\prime}$ loop by allowing the $Z^{\prime}$ mass to be free and setting large $\left|\sin \theta_{G}\right|=\mathrm{O}\left(10^{-1}\right)$, which is obtained when $g_{y}^{\prime}$ and $g_{z}^{\prime}$ are $\mathrm{O}\left(10^{-1}\right)$. This corresponds to $M_{Z^{\prime}}=\mathrm{O}\left(M_{Z}\right)$, which is, of course, excluded. Yet, even in this case, the correction from the $Z$ and $Z^{\prime}$ loops is small and has the same order of magnitude, $\mathrm{O}\left(10^{-7}\right) \mathrm{eV}$. Thus, the individual contributions from beyond SM loops cannot be significantly larger than the SM contributions.

\section{CONCLUSIONS}

In this paper, we have computed the one-loop corrections to the mass matrix of the active neutrinos in a gauged $\mathrm{U}(1)$ extension of the standard model of particle interactions. The field content of the model consists of a new complex scalar field and three right-handed neutrinos-sterile under the standard model interactions - in addition to the fields in the standard model and the new gauge field. The neutrino masses are generated by Dirac- and Majorana-type Yukawa terms, which after spontaneous symmetry breaking of both scalar fields give rise to neutrino masses in the way of the type I seesaw mass generation. We have used the $R_{\xi}$ gauge and have shown that the one-loop corrections are (i) independent of the gauge fixing parameters, (ii) finite, and (iii) independent of the regularization scale. We have also demonstrated how the formula for the one-loop mass corrections can be generalized to the case of an arbitrary number of new U(1) groups, complex scalars, and righthanded neutrinos.

We have provided a numerical estimate of the size of the mass corrections in the context of the superweak model, in which the new neutral gauge boson $Z^{\prime}$ is much lighter than the $Z$ boson of the standard model. We have found that in the mass range of $M_{Z^{\prime}} \in[20,200] \mathrm{MeV}$, motivated by a possible explanation of the relic density of dark matter in the Universe, the relative mass corrections to the treelevel mass matrix elements do not exceed the per mill level. Hence, the model is stable against higher-order corrections in the neutrino sector, which motivates further studies to explore the viable parameter space of the model regarding the mixings between the active and sterile neutrinos [31].

\section{ACKNOWLEDGMENTS}

We are grateful to Josu Hernández-García for discussions on this project. This work was supported by Grant No. K 125105 of the National Research, Development and Innovation Fund in Hungary.

\section{APPENDIX A: SOME PROPERTIES OF NEUTRINO MASS AND MIXING MATRICES}

In this Appendix, we derive some useful relations among the neutrino mass and mixing matrices. The matrix $\mathbf{U}$ that diagonalizes the neutrino mass matrix is unitary, hence,

$$
\begin{aligned}
\mathbf{U}^{\dagger} & =\left(\begin{array}{l}
\mathbf{U}_{L} \\
\mathbf{U}_{R}^{*}
\end{array}\right)\left(\mathbf{U}_{L}^{\dagger}, \mathbf{U}_{R}^{T}\right)=\left(\begin{array}{ll}
\mathbf{U}_{L} \mathbf{U}_{L}^{\dagger} & \mathbf{U}_{L} \mathbf{U}_{R}^{T} \\
\mathbf{U}_{R}^{*} \mathbf{U}_{L}^{\dagger} & \mathbf{U}_{R}^{*} \mathbf{U}_{R}^{T}
\end{array}\right) \\
& =\left(\begin{array}{ll}
\mathbf{1}_{3} & \mathbf{0}_{3} \\
\mathbf{0}_{3} & \mathbf{1}_{3}
\end{array}\right),
\end{aligned}
$$

from which we obtain the following important relations:

$$
\mathbf{U}_{L} \mathbf{U}_{L}^{\dagger}=\mathbf{1}_{3}, \quad \mathbf{U}_{R} \mathbf{U}_{R}^{\dagger}=\mathbf{1}_{3},
$$

and

$$
\mathbf{U}_{L} \mathbf{U}_{R}^{T}=\mathbf{U}_{R}^{*} \mathbf{U}_{L}^{\dagger}=\mathbf{0}_{3},
$$

where $\mathbf{1}_{n}$ denotes the $n \times n$ unit matrix. The second unitarity conditions gives

$\mathbf{U}^{\dagger} \mathbf{U}=\left(\mathbf{U}_{L}^{\dagger}, \mathbf{U}_{R}^{T}\right)\left(\begin{array}{c}\mathbf{U}_{L} \\ \mathbf{U}_{R}^{*}\end{array}\right)=\mathbf{U}_{L}^{\dagger} \mathbf{U}_{L}+\mathbf{U}_{R}^{T} \mathbf{U}_{R}^{*}=\mathbf{1}_{6}$.

Using Eq. (2.35), we derive

$$
\begin{aligned}
\mathbf{U}_{L}^{*} \mathbf{M} \mathbf{U}_{L}^{\dagger}= & \mathbf{U}_{L}^{*}\left(\mathbf{U}_{L}^{T}, \mathbf{U}_{R}^{\dagger}\right)\left(\begin{array}{cc}
\mathbf{0}_{3} & \mathbf{M}_{D}^{T} \\
\mathbf{M}_{D} & \mathbf{M}_{N}
\end{array}\right)\left(\begin{array}{c}
\mathbf{U}_{L} \\
\mathbf{U}_{R}^{*}
\end{array}\right) \mathbf{U}_{L}^{\dagger} \\
= & \mathbf{U}_{L}^{*} \mathbf{U}_{L}^{T} \mathbf{M}_{D} \mathbf{U}_{R}^{*} \mathbf{U}_{L}^{\dagger}+\mathbf{U}_{L}^{*} \mathbf{U}_{R}^{\dagger} \mathbf{M}_{D} \mathbf{U}_{L} \mathbf{U}_{L}^{\dagger} \\
& +\mathbf{U}_{L}^{T} \mathbf{U}_{R}^{\dagger} \mathbf{M}_{N} \mathbf{U}_{R}^{*} \mathbf{U}_{L}^{\dagger},
\end{aligned}
$$

and then with relations in Eq. (A3), we obtain

$$
\mathbf{U}_{L}^{*} \mathbf{M} \mathbf{U}_{L}^{\dagger}=\mathbf{0}_{3} .
$$

Analogous calculations yield

$$
\mathbf{U}_{R} \mathbf{M} \mathbf{U}_{L}^{\dagger}=\mathbf{M}_{D} .
$$

Multiplying Eq. (A6) with $\mathbf{U}_{R}^{\dagger}$ from the left and using Eq. (A4), we find

$$
\begin{aligned}
\mathbf{U}_{R}^{\dagger} \mathbf{M}_{D} & =\mathbf{U}_{R}^{\dagger} \mathbf{U}_{R} \mathbf{M} \mathbf{U}_{L}^{\dagger}=\left(\mathbf{1}_{6}-\mathbf{U}_{L}^{T} \mathbf{U}_{L}^{*}\right) \mathbf{M} \mathbf{U}_{L}^{\dagger} \\
& =\mathbf{M} \mathbf{U}_{L}^{\dagger}-\mathbf{U}_{L}^{T} \mathbf{U}_{L}^{*} \mathbf{M} \mathbf{U}_{L}^{\dagger},
\end{aligned}
$$

where the last term vanishes by Eq. (A5), so

$$
\mathbf{U}_{R}^{\dagger} \mathbf{M}_{D}=\mathbf{M} \mathbf{U}_{L}^{\dagger} .
$$

Finally, 


$$
\begin{aligned}
\mathbf{U}_{R} \mathbf{M} \mathbf{U}_{R}^{T} & =\mathbf{U}_{R}\left(\mathbf{U}_{L}^{T}, \mathbf{U}_{R}^{\dagger}\right)\left(\begin{array}{cc}
\mathbf{0} & \mathbf{M}_{D}^{T} \\
\mathbf{M}_{D} & \mathbf{M}_{N}
\end{array}\right)\left(\begin{array}{c}
\mathbf{U}_{L} \\
\mathbf{U}_{R}^{*}
\end{array}\right) \mathbf{U}_{R}^{T} \\
& =\mathbf{U}_{R}\left(\mathbf{U}_{L}^{T} \mathbf{M}_{D}^{T} \mathbf{U}_{R}^{*}+\mathbf{U}_{R}^{\dagger} \mathbf{M}_{D} \mathbf{U}_{L}+\mathbf{U}_{R}^{\dagger} \mathbf{M}_{N} \mathbf{U}_{R}^{*}\right) \mathbf{U}_{R}^{T} .
\end{aligned}
$$

Expanding the factors into the parentheses, the first two terms give vanishing contribution by Eq. (A4), while utilizing Eq. (A2), the last one is simply $\mathbf{M}_{N}$, so

$$
\mathbf{U}_{R} \mathbf{M U}_{R}^{T}=\mathbf{M}_{N}
$$

Now Eq. (A4) allows us to derive

$$
\begin{aligned}
\mathbf{U}_{R}^{\dagger} \mathbf{M}_{N} & =\mathbf{U}_{R}^{\dagger} \mathbf{U}_{R} \mathbf{M} \mathbf{U}_{R}^{T}=\left(\mathbf{1}_{6}-\mathbf{U}_{L}^{T} \mathbf{U}_{L}^{*}\right) \mathbf{M} \mathbf{U}_{R}^{T} \\
& =\mathbf{M} \mathbf{U}_{R}^{T}-\mathbf{U}_{L}^{T} \mathbf{U}_{L}^{*} \mathbf{M} \mathbf{U}_{R}^{T},
\end{aligned}
$$

where the second term on the right does not vanish this time.

\section{APPENDIX B: EVALUATION OF THE VECTOR BOSON EXCHANGE DIAGRAM}

The vector boson exchange diagrams shown in the bottom row of Fig. 1 contribute gauge-dependent terms to the neutrino self-energy. In order to show that the gaugedependent terms cancel once contributions from all particles are considered, it is useful to eliminate the loop momentum from the one-loop integral corresponding to the vector boson exchange diagram. A decomposition to achieve this was used in Ref. [41], and we shall derive it here as well. In Ref. [42], Eq (4.4) contains the selfenergy,

$\mathrm{i} \Sigma_{V}(p)=-\int \frac{d^{d} \ell}{(2 \pi)^{d}} \boldsymbol{\Gamma}^{\mu \dagger} \mathbf{P}(p-\ell) \boldsymbol{\Gamma}^{\nu} P_{\mu \nu}\left(\ell, M_{V}^{2} ; \xi_{V}\right)$,

where the $6 \times 6$ matrices are defined as follows. The matrix $\mathbf{P}$ is the fermion propagator, diagonal in the mass eigenstates,

$$
\begin{array}{rlrl}
\mathbf{P}(p-\ell) & =[(\not p-\ell) \mathbf{1}-\mathbf{M}]^{-1}, & \text { while } & \boldsymbol{\Gamma}^{\mu}=-\mathrm{i} e \gamma^{\mu} \mathbf{A}, \\
\text { with } \quad \mathbf{A} & =\boldsymbol{\Gamma}^{L} P_{L}+\boldsymbol{\Gamma}^{R} P_{R} . & \text { (B2) }
\end{array}
$$

In the following, we shall write $\mathbf{P}$ for $\mathbf{P}(p-\ell)$. The matrix $\mathbf{A}$ is self-adjoint, $\mathbf{A}^{\dagger}=\mathbf{A}$, and so is $\boldsymbol{\Gamma}^{L / R}$. We also introduce the abbreviation

$$
\tilde{\mathbf{A}}=\Gamma^{R} P_{L}+\Gamma^{L} P_{R},
$$

which will simplify our calculations. In order to compute the loop integral easily containing the neutral vector boson propagator in the neutrino self-energy loop, in this Appendix we perform tensor reduction of the matrix product $\ell \mathbf{A}^{\dagger} \mathbf{P} \ell \mathbf{A}$

such that the numerator factor be at most linear in the loop momentum $\ell$.

When the fermion momentum $p$ appears as $\not p$ at the extreme left or right of the expression, it satisfies the Dirac equation $\not p \mathbf{1}_{6}=\mathbf{M}$ (both Dirac and Majorana fermions do so); thus, we can replace formally $\not p$ with $\mathbf{M}$. Let us first write the identity

$$
\ell \mathbf{A}^{\dagger}=(\ell \ell-\not p+\mathbf{M}) \mathbf{A}^{\dagger}=\mathbf{M} \mathbf{A}^{\dagger}-(\not \not-\not \ell) \mathbf{A}^{\dagger} .
$$

The chiral coupling matrix A anticommutes with the Dirac matrices $\gamma_{\mu}$, hence,

$$
\begin{aligned}
\ell \mathbf{A}^{\dagger} & =\mathbf{M} \mathbf{A}^{\dagger}-\tilde{\mathbf{A}}^{\dagger}((\not \not-\ell) \mathbf{1}-\mathbf{M}+\mathbf{M}) \\
& =\mathbf{M} \mathbf{A}^{\dagger}-\tilde{\mathbf{A}}^{\dagger} \mathbf{P}^{-1}-\tilde{\mathbf{A}} \tilde{\mathbf{A}}^{\dagger} \mathbf{M},
\end{aligned}
$$

and similarly,

$$
\mathbf{P} \ell \mathbf{A}=-\mathbf{A}-\mathbf{P M A}+\mathbf{P} \tilde{\mathbf{A}} \mathbf{M} .
$$

Multiplying Eqs. (B6) and (B7), we obtain the expression (B4), and its expansion yields

$$
\begin{aligned}
\ell \mathbf{A}^{\dagger} \mathbf{P} \ell \mathbf{A}= & -\mathbf{M A}^{\dagger} \mathbf{A}-\mathbf{M} \mathbf{A}^{\dagger} \mathbf{P M A}+\mathbf{M A}^{\dagger} \mathbf{P A M} \\
& +\tilde{\mathbf{A}}^{\dagger} \mathbf{P}^{-1} \mathbf{A}+\tilde{\mathbf{A}}^{\dagger} \mathbf{M A}-\tilde{\mathbf{A}}^{\dagger} \tilde{\mathbf{A}} \mathbf{M}+\tilde{\mathbf{A}}^{\dagger} \mathbf{M A} \\
& +\tilde{\mathbf{A}}^{\dagger} \mathbf{M P M A}-\tilde{\mathbf{A}}^{\dagger} \mathbf{M P A} \mathbf{A} \mathbf{M} .
\end{aligned}
$$

Using that $\not p \mathbf{A}=\tilde{\mathbf{A}} \not p$, the fourth term can rearranged as

$$
\begin{aligned}
\tilde{\mathbf{A}}^{\dagger} \mathbf{P}^{-1} \mathbf{A} & =\tilde{\mathbf{A}}^{\dagger}((\not p-\ell) \mathbf{1}-\mathbf{M}) \mathbf{A} \\
& =\frac{1}{2} \tilde{\mathbf{A}}^{\dagger} \not p \mathbf{A}+\frac{1}{2} \tilde{\mathbf{A}}^{\dagger} \not p \mathbf{A}-\tilde{\mathbf{A}}^{\dagger} \not \mathbf{A}-\tilde{\mathbf{A}}^{\dagger} \mathbf{M} \mathbf{A} \\
& =\frac{1}{2} \not p \mathbf{A}^{\dagger} \mathbf{A}+\frac{1}{2} \tilde{\mathbf{A}}^{\dagger} \tilde{\mathbf{A}} \not p-\tilde{\mathbf{A}}^{\dagger} \ell \mathbf{A}-\tilde{\mathbf{A}}^{\dagger} \mathbf{M} \mathbf{A} .
\end{aligned}
$$

The $\not p$ is on extreme left and right; hence, can be replaced with M, giving

$\tilde{\mathbf{A}}^{\dagger} \mathbf{P}^{-1} \mathbf{A}=\frac{1}{2} \mathbf{M} \mathbf{A}^{\dagger} \mathbf{A}+\frac{1}{2} \tilde{\mathbf{A}}^{\dagger} \tilde{\mathbf{A}} \mathbf{M}-\tilde{\mathbf{A}}^{\dagger} \ell \mathbf{A}-\tilde{\mathbf{A}}^{\dagger} \mathbf{M} \mathbf{A}$.

Substituting Eq. (B10) into Eq. (B8), we obtain

$$
\ell \mathbf{A}^{\dagger} \mathbf{P} \ell \mathbf{A}=\mathbf{M}_{0}+\mathbf{M}_{1}+\mathbf{M}_{2},
$$

where we introduced the abbreviations

$$
\begin{aligned}
& \mathbf{M}_{0}=-\tilde{\mathbf{A}}^{\dagger} \mathscr{\ell} \mathbf{A}, \\
& \mathbf{M}_{1}=-\frac{1}{2} \mathbf{M} \mathbf{A}^{\dagger} \mathbf{A}-\frac{1}{2} \tilde{\mathbf{A}}^{\dagger} \tilde{\mathbf{A}} \mathbf{M}+\tilde{\mathbf{A}}^{\dagger} \mathbf{M} \mathbf{A},
\end{aligned}
$$


and

$$
\begin{aligned}
\mathbf{M}_{2}= & -\mathbf{M A}^{\dagger} \mathbf{P M A}+\mathbf{M A}^{\dagger} \mathbf{P} \tilde{\mathbf{A}} \mathbf{M} \\
& +\tilde{\mathbf{A}}^{\dagger} \mathbf{M P M A}-\tilde{\mathbf{A}}^{\dagger} \mathbf{M P} \tilde{\mathbf{A}} \mathbf{M} \\
= & \left(\mathbf{M A}^{\dagger}-\tilde{\mathbf{A}}^{\dagger} \mathbf{M}\right) \mathbf{P}(\tilde{\mathbf{A}} \mathbf{M}-\mathbf{M A}),
\end{aligned}
$$

which correspond to constant, linear, and quadratic terms in the neutrino mass matrix $\mathbf{M}$. We now discuss the contribution from each term in Eq. (B11) separately.

The first constant term gives vanishing contribution to the loop integral as it is odd in the loop momentum. The other two terms can be decomposed into left- and rightchiral pieces:

$$
\mathbf{M}_{i}=\mathbf{M}_{i}^{L} P_{L}+\mathbf{M}_{i}^{R} P_{R} .
$$

Our goal is to compute the one-loop correction (3.7) to the tree-level mass matrix of the light neutrinos. In order to obtain it, one sandwiches the left-handed pieces $\mathbf{M}_{i}^{L}$ between the matrices $\mathbf{U}_{L}^{*}$ and $\mathbf{U}_{L}^{\dagger}$. Using the properties of the neutrino mixing matrices of Appendix A, we immediately see that

$$
\mathbf{U}_{L}^{*} \mathbf{M}_{1}^{L} \mathbf{U}_{L}^{\dagger}=0,
$$

while lengthy computations yield

$$
\begin{aligned}
\mathbf{U}_{L}^{*} \mathbf{M}_{2}^{L} \mathbf{U}_{L}^{\dagger}= & -\left(C_{V \nu \nu}^{L}-C_{V \nu \nu}^{R}\right)^{2} \mathbf{U}_{L}^{*} \mathbf{M P M} \mathbf{U}_{L}^{\dagger} \\
& + \text { terms that do not contribute to } \mathbf{B}_{L}(0) .
\end{aligned}
$$

Here we outline the steps needed to reach Eq. (B16).

First, in order to find the left-chiral part $\mathbf{M}_{2}^{L}$, we substitute $\mathbf{A}$ and $\tilde{\mathbf{A}}$ into Eq. (B13). We write the denominator of the fermion propagator as

$$
(\mathbf{P})_{i j}=\delta_{i j} \frac{\not p-\not \subset+m_{i}}{(p-\ell)^{2}-m_{i}^{2}},
$$

and use the following relations for the Dirac projectors:

$$
\begin{aligned}
& P_{L / R}(q+m) P_{L / R}=m P_{L / R}, \\
& P_{L / R}(q+m) P_{R / L}=\not q P_{R / L}
\end{aligned}
$$

valid for any momentum $q$ and mass $m$. Hence,

$$
\begin{aligned}
P_{L / R}(\mathbf{P})_{i j} P_{L / R} & =\left((\mathbf{P})_{i j}-\delta_{i j} \frac{\not p-\not 1}{(p-\ell)^{2}-m_{i}^{2}}\right) P_{L / R}, \\
P_{L / R}(\mathbf{P})_{i j} P_{R / L} & =\delta_{i j} \frac{\not p-\not 1}{(p-\ell)^{2}-m_{i}^{2}} P_{R / L},
\end{aligned}
$$

and therefore, we obtain

$$
\begin{aligned}
\mathbf{M}_{2}^{L}= & -\mathbf{M} \Gamma^{L \dagger} \mathbf{P M} \Gamma^{L}+\mathbf{M} \Gamma^{L \dagger} \mathbf{P} \Gamma^{R} \mathbf{M}+\Gamma^{R \dagger} \mathbf{M P M} \Gamma^{L} \\
& -\Gamma^{R \dagger} \mathbf{M P} \Gamma^{R} \mathbf{M}+\mathbf{D},
\end{aligned}
$$

where the last term is proportional to $(\not \not-\ell \ell)$ :

$$
\begin{aligned}
\mathbf{D}= & {\left.\left[\mathbf{M}\left(\boldsymbol{\Gamma}^{L^{\dagger}}-\boldsymbol{\Gamma}^{R_{\dagger}}\right)-\left(\boldsymbol{\Gamma}^{L \dagger}-\boldsymbol{\Gamma}^{R_{\dagger}}\right) \mathbf{M}\right)\right](\not \not-\not) } \\
& \times\left[(p-l)^{2} \mathbf{1}_{6}-\mathbf{M}^{2}\right]^{-1}\left(\boldsymbol{\Gamma}^{R} \mathbf{M}-\mathbf{M} \boldsymbol{\Gamma}^{L}\right) .
\end{aligned}
$$

Then using the matrix relations derived in Appendix A, we can compute the following identities:

$$
\begin{array}{r}
\mathbf{U}_{L}^{*} \mathbf{M} \boldsymbol{\Gamma}^{L^{\dagger}}=-C_{V \nu \nu}^{R} \mathbf{U}_{L}^{*} \mathbf{M}, \quad \mathbf{U}_{L}^{*} \boldsymbol{\Gamma}^{R \dagger}=-C_{V \nu \nu}^{L} \mathbf{U}_{L}^{*}, \\
\boldsymbol{\Gamma}^{R} \mathbf{M} \mathbf{U}_{L}^{\dagger}=C_{V \nu \nu}^{R} \mathbf{M} \mathbf{U}_{L}^{\dagger}, \quad \boldsymbol{\Gamma}^{L} \mathbf{U}_{L}^{\dagger}=C_{V \nu \nu}^{L} \mathbf{U}_{L}^{\dagger} .
\end{array}
$$

Finally, sandwiching Eq. (B20) gives us

$$
\begin{aligned}
\mathbf{U}_{L}^{*} \mathbf{M}_{2}^{L} \mathbf{U}_{L}^{\dagger}= & -\left(C_{V \nu \nu}^{L}-C_{V \nu \nu}^{R}\right)^{2} \mathbf{U}_{L}^{*} \mathbf{M P M} \mathbf{U}_{L}^{\dagger} \\
& +\mathbf{U}_{L}^{*} \mathbf{D} \mathbf{U}_{L}^{\dagger} .
\end{aligned}
$$

As mentioned, the last term is proportional to $(\not p-\ell)$, but only the term with $\ell$ contributes to $\mathbf{B}_{L}(p=0)$. That piece being an odd function of $\ell$ vanishes upon integration, which completes the proof of Eq. (B16).

The charged vector bosons $W^{ \pm}$also contribute to the neutrino self-energy. The corresponding Feynman rules are

$\Gamma_{W^{-} \bar{\ell}_{\nu}}^{\mu}=-\mathrm{i} e \gamma^{\mu} \boldsymbol{\Gamma}_{W^{-} \bar{\ell}_{\nu}}^{L} P_{L}, \quad \boldsymbol{\Gamma}_{W^{+} \nu \ell}^{\mu}=-\mathrm{i} e \gamma^{\mu} \boldsymbol{\Gamma}_{W^{+} \nu \ell}^{L} P_{L}$,

where

$$
\boldsymbol{\Gamma}_{W^{-} \bar{\ell}_{\nu}}^{L}=C_{W \ell \nu}\left(\mathbf{U}_{L}^{\ell} \mathbf{U}_{L}\right)_{i j},
$$

with $\mathbf{U}_{L}^{\ell}$ being the charged lepton mixing matrix and $\boldsymbol{\Gamma}_{W^{-} \bar{\ell}_{\nu}}^{L}=\left(\boldsymbol{\Gamma}_{W^{+} \nu \ell}^{L}\right)^{\dagger}$. The charged vector boson contribution to (3.7) is proportional to $\mathbf{U}_{L}^{*} \mathbf{M} \mathbf{U}_{L}^{\dagger}$, which vanishes identically as shown in Appendix A. 
[1] Y. Fukuda et al., Measurements of the Solar Neutrino Flux from Super-Kamiokande's First 300 Days, Phys. Rev. Lett. 81, 1158 (1998).

[2] Q. R. Ahmad et al., Measurement of the Rate of $\nu_{e}+d \rightarrow$ $p+p+e^{-}$Interactions Produced by ${ }^{8} b$ Solar Neutrinos at the Sudbury Neutrino Observatory, Phys. Rev. Lett. 87, 071301 (2001).

[3] H. Fritzsch, M. Gell-Mann, and P. Minkowski, Vectorlike weak currents and new elementary fermions, Phys. Lett. 59B, 256 (1975).

[4] P. Minkowski, $\mu \rightarrow e \gamma$ at a rate of one out of $10^{9}$ muon decays?, Phys. Lett. 67B, 421 (1977).

[5] M. Gell-Mann, P. Ramond, and R. Slansky, Complex spinors and unified theories, Conf. Proc. C 790927, 315 (1979).

[6] T. Yanagida, Horizontal symmetry and masses of neutrinos, Conf. Proc. C 7902131, 95 (1979).

[7] R. N. Mohapatra and G. Senjanović, Neutrino Mass and Spontaneous Parity Nonconservation, Phys. Rev. Lett. 44, 912 (1980).

[8] J. Schechter and J.W.F. Valle, Neutrino masses in $\mathrm{SU}(2) \times \mathrm{U}(1)$ theories, Phys. Rev. D 22, 2227 (1980).

[9] M. Magg and C. Wetterich, Neutrino mass problem and gauge hierarchy, Phys. Lett. 94B, 61 (1980).

[10] S. L. Glashow, The future of elementary particle physics, NATO Sci. Ser. B 61, 687 (1980).

[11] B. Abi et al., Measurement of the Positive Muon Anomalous Magnetic Moment to 0.46 ppm, Phys. Rev. Lett. 126, 141801 (2021).

[12] A. A. Aguilar-Arevalo et al., Significant Excess of Electronlike Events in the MiniBooNE Short-Baseline Neutrino Experiment, Phys. Rev. Lett. 121, 221801 (2018).

[13] A. E. Nelson and J. Walsh, Short baseline neutrino oscillations and a new light gauge boson, Phys. Rev. D 77, 033001 (2008).

[14] J. Heeck and W. Rodejohann, Gauged $L_{\mu}-L_{\tau}$ and different muon neutrino and anti-neutrino oscillations: MINOS and beyond, J. Phys. G 38, 085005 (2011).

[15] E. Ma, Gauged $B-3 L_{\tau}$ and radiative neutrino masses, Phys. Lett. B 433, 74 (1998).

[16] E. Ma, D. P. Roy, and S. Roy, Gauged $L_{\mu}-L_{\tau}$ with large muon anomalous magnetic moment and the bimaximal mixing of neutrinos, Phys. Lett. B 525, 101 (2002).

[17] K. Asai, Predictions for the neutrino parameters in the minimal model extended by linear combination of $\mathrm{U}(1)_{L_{e}-L_{u}}$, $\mathrm{U}(1)_{L_{\mu}-L_{\tau}}$ and $\mathrm{U}(1)_{B-L}$ gauge symmetries, Eur. Phys. J. C 80, 76 (2020).

[18] D. Bhatia, S. Chakraborty, and A. Dighe, Neutrino mixing and $R_{K}$ anomaly in $\mathrm{U}(1)_{X}$ models: A bottom-up approach, J. High Energy Phys. 03 (2017) 117.

[19] R. Adhikari, J. Erler, and E. Ma, Seesaw neutrino mass and new U(1) gauge symmetry, Phys. Lett. B 672, 136 (2009).

[20] D. Borah, L. Mukherjee, and S. Nandi, Low scale $\mathrm{U}(1)_{X}$ gauge symmetry as an origin of dark matter, neutrino mass and flavour anomalies, J. High Energy Phys. 12 (2020) 052.

[21] A. Pilaftsis, Radiatively induced neutrino masses and large Higgs neutrino couplings in the standard model with Majorana fields, Z. Phys. C 55, 275 (1992).
[22] D. A. Sierra and C. E. Yaguna, On the importance of the 1-loop finite corrections to seesaw neutrino masses, J. High Energy Phys. 08 (2011) 013.

[23] J. Lopez-Pavon, S. Pascoli, and C.-f. Wong, Can heavy neutrinos dominate neutrinoless double beta decay?, Phys. Rev. D 87, 093007 (2013).

[24] J. Lopez-Pavon, E. Molinaro, and S. T. Petcov, Radiative corrections to light neutrino masses in low scale type I seesaw scenarios and neutrinoless double beta decay, J. High Energy Phys. 11 (2015) 030.

[25] P. Ballett, M. Hostert, and S. Pascoli, Neutrino masses from a dark neutrino sector below the electroweak scale, Phys. Rev. D 99, 091701 (2019).

[26] W. Grimus and L. Lavoura, One-loop corrections to the seesaw mechanism in the multi-Higgs-doublet standard model, Phys. Lett. B 546, 86 (2002).

[27] W. Grimus and M. Löschner, Renormalization of the multiHiggs-doublet Standard Model and one-loop lepton mass corrections, J. High Energy Phys. 11 (2018) 087.

[28] V. Dūdènas and T. Gajdosik, Gauge dependence of tadpole and mass renormalization for a seesaw extended 2HDM, Phys. Rev. D 98, 035034 (2018).

[29] A. Denner, L. Jenniches, J.-N. Lang, and C. Sturm, Gaugeindependent $\overline{\mathrm{MS}}$ renormalization in the 2HDM, J. High Energy Phys. 09 (2016) 115.

[30] Z. Trócsányi, Super-weak force and neutrino masses, Symmetry 12, 107 (2020).

[31] T. J. Kärkkäinen and Z. Trócsányi, Experimental constraints on the neutrino and gauge parameters of the super-weak $\mathrm{U}$ (1) extension of the standard model, arXiv:2105.13360.

[32] S. Iwamoto, K. Seller, and Z. Trócsányi, Sterile neutrino dark matter in a $\mathrm{U}(1)$ extension of the standard model, arXiv:2104.11248.

[33] Z. Péli, I. Nándori, and Z. Trócsányi, Particle physics model of curvaton inflation in a stable universe, Phys. Rev. D 101, 063533 (2020).

[34] F. Staub, From superpotential to model files for FEYNARTS and CAlChep/COMPHeP, Comput. Phys. Commun. 181, 1077 (2010).

[35] F. Staub, Automatic calculation of supersymmetric renormalization group equations and loop corrections, Comput. Phys. Commun. 182, 808 (2011).

[36] F. Staub, SARAH 4: A tool for (not only SUSY) model builders, Comput. Phys. Commun. 185, 1773 (2014).

[37] See Supplemental Material at http://link.aps.org/ supplemental/10.1103/PhysRevD.104.055042, for SARAH model files of the superweak model, used to generate Feynman rules.

[38] Note the opposite sign convention for $\theta_{Z}$ in this work and in Ref. [30] where this mixing angle was denoted as $\theta_{T}$, so $\theta_{Z}=-\theta_{T}$

[39] This matrix coincides with the unitary matrix $U^{V, \dagger}$ used by SARAH.

[40] Z. Péli and Z. Trócsányi, Stability of the vacuum as constraint on $U(1)$ extensions of the standard model, arXiv:1902.02791.

[41] M. Löschner, Renormalization and one-loop corrections of lepton masses, Ph.D. thesis, Vienna University, 2018.

[42] S. Weinberg, Perturbative calculations of symmetry breaking, Phys. Rev. D 7, 2887 (1973). 